\title{
Life Expectancy, Human Capital, Social Security and Growth
}

\author{
Cruz A. Echevarría*, Amaia Iza \\ (The Basque Country University)
}

April 2006

\begin{abstract}
We analyze the effects of changes in the mortality rate upon life expectancy, education, retirement age, human capital and growth in the presence of social security. We build a vintage growth, overlapping generations model in which individuals choose the time length of education and retirement age, and where unfunded social security pensions depend on workers' past contributions. Social security has a positive effect on education, but pension benefits favor reductions in retirement age. The net effect is that starting from a benchmark case, higher life expectancies give rise to lower growth rates in the presence of social security as the share of active population is reduced. In addition, higher social security contribution rates reduce the growth rate.
\end{abstract}

JEL Classification Numbers: O40, H55, J10

KEY WORDS: Mortality Rate, Social Security, Growth

* Corresponding author: Cruz A. Echevarría. Departamento de Fundamentos del Análisis Económico II. Facultad de Ciencias Económicas y Empresariales. Universidad del País Vasco. Avda. Lehendakari Aguirre 83. 48015 Bilbao (SPAIN). Tel. (34) 946013775. Fax (34) 946013774. Email: cruz.echeva1ria@ehu.es 


\section{Introduction}

This article intends to integrate two streams of the economic literature generally considered separately: i) life expectancy and endogenous growth; and ii) aging, unfunded social security and exogenous economic growth.

The relationship between life expectancy at birth and per capita growth has been largely studied both empirically and theoretically. Regarding the empirical evidence, the hypothesis that reductions in mortality rates have a non monotonic relationship with per growth rates is mostly supported. By using time series data, Rodriguez et al. (1999) find a positive effect of life expectancy upon GDP growth rate in Venezuela in 1970-90. However, Malmberg (1994) finds a negative relationship in Sweden in 1950-89. Analysis of cross section data also shows that this relationship is not monotonic. Preliminary data from Latin America and Caribbean countries indicate that $G D P$ growth is positively associated with life expectancy. [See World Health Organization (1999), Box 1.2, p. 9.] Barro et al. (1995), using a sample of 97 countries, estimate that an increment in life expectancy of 13 years would increase per capita growth rate by $1.4 \%$ per year. Zhang et al. (2005) shows a clear, positive relationship, but at a diminishing rate. Finally, some other studies have found mixed evidence: increases in life expectancy have paralleled higher growth rates for low life expectancies, but lower growth rates for high life expectancies. [See Zhang et al. (2003) and references therein.]

Regarding theoretical works, they mostly assume that human capital accumulation is the engine of growth. Some of them conclude that the relationship is always positive, whereas some others are able to obtain an inverted $U$ pattern. Among the former we could mention Ehrlich et al. (1991) and Hu (1999). In this type of models, a higher life expectancy increases the period length in which the return to human capital investment is obtained, thus allowing for higher rates of return, and, 
in consequence, higher investment and growth rates. ${ }^{1}$

Some other works have obtained an inverted $U$ pattern between life expectancy and per capita growth what is consistent with the mixed empirical evidence above mentioned (both with historical and cross-section data). De la Croix et al. (1999) build up an economy where the effect of a reduction in the mortality rate upon the duration of education is such that the growth rate becomes higher for high mortality rates (as in underdeveloped countries), but lower for low mortality rates (as in industrialized countries). The same result is obtained in Boucekkine et al. (2002), where they assume uncertain lifetime horizon and endogenous retirement age. In both papers, labor force is the unique input in production, and the intuition behind the negative sloped part is that average human capital of the labor force becomes more obsolete as life expectancy increases. ${ }^{2}$ Zhang and Zhang (2003) and Zhang et al. (2003) also obtain this result but by means of a different channel: not through own education time, but through the expenditure on children's education.

Regarding the second line of research mentioned in the beginning of the section, a considerable number of articles in the recent literature deal with the connections between population aging, pay-as-you-go social security and retirement age. One recurring subject in this literature is the effect of social security upon workers' voluntary retirement age. Along these lines, the available empirical evidence suggests that, at least for the US economy, social security is relevant for retirement age issues, even though there is no total agreement on the effects of variations in the generosity of the social security program. [See, e.g., Diamond et al. (1997) and Coile et al. (2000).]

\footnotetext{
${ }^{1}$ Echevarría (2003) shows in an exogenous retirement model in which human capital investment depends positively on the number of remaining active periods until the individual's retirement that increases in life expectancy give rise to higher growth rates only if accompanied by simultaneous exogenous increments in the active period (i.e., delays in the retirement age).

${ }^{2}$ Building on Boucekkine et al. (2002), but allowing for physical capital along with human capital in a certain lifetime horizon, Echevarría (2004) obtains the same relationship.
} 
In this article we study to what extent introducing unfunded social security affects the relationship between life expectancy and per capita growth rate, taking into account its effects on education and retirement age incentives. Our starting point is Boucekkine et al. (2002). It is, in essence, an overlapping generations model with uncertain, finite lifetime horizon. Fertility and mortality rates are exogenous, and individuals choose their optimal education time length and retirement age, thereby way influencing average human capital in a vintage way and the economy's growth. In our extension we include an unfunded social security system whose pension benefits depend on the contributions made by workers during their active periods. According to this design, social security will influence not only individual decisions (namely, years of education and retirement age), but also aggregate magnitudes such as economic growth.

Why might the inclusion of social security be of any interest? In such a setup the return to human capital investment is not constrained to labor income while active, but also extends to pensions during retirement, which are in turn related to past wage earnings. Therefore, when individuals choose their optimal length of the education period, they take into account not only the effect on future labor earnings, but also on future pension benefits. Additionally, voluntary retirement age will also depend on the incentives that the public pension system embeds. As a consequence, we have that social security will affect the size of the working population and also that of the aggregate human capital in the economy. Therefore, social security will influence the response of the economy's growth rate after, say, a fall in the mortality rate and the corresponding increase in the life expectancy.

This article contains two different parts. In part one we solve analytically the individual problem (individuals and firms), the steady state per capita growth rate and the social security budget balance. We characterize the parameter space which 
determines the type of solution for the individuals' problem and prove the existence and the uniqueness of that solution. Furthermore, we prove the existence and uniqueness of steady state per capita growth rate and social security balance for the case of interior solutions for education and retirement age.

In part two, given that the system of equations that solves the model turns out to be non-linear, explicit solutions and analytical results are excluded. Thus, we limit our results to numerical solutions to comparative statics exercises between steady states. We compare two scenarios (with and without unfunded social security). We are able to replicate the observed inverted $U$ relationship between life expectancy and per capita growth in our scenario with social security. Our main finding is that introducing social security affects the incentives to education time and early retirement in such a way that the major force driving the negatively sloped part of that locus is the fall of the share of active population (i.e. workers), not the obsolescence of human capital among workers as it is the case when there is no social security.

The rest of the article is organized as follows. Section 2 shows the main demographic features of our model. Readers interested only in the economic aspects may proceed to the next Section. Section 3 introduces the economic model: the individual problem, the aggregate technology of production, the optimal education and retirement age, the aggregates, the social security balance and the balanced growth path. The numerical example and the results are shown in Section 4. Section 5 shows the conclusions. A mathematical Appendix contains the formal proofs. 


\section{Demographic structure}

Individuals face an uncertain lifetime horizon with a positive, age increasing instantaneous mortality rate, so that there exists a maximum attainable age. ${ }^{3}$

More precisely, the demographic structure in this economy is characterized by the survival rate distribution

$$
m(a)=\frac{\alpha-e^{-\beta a}}{\alpha-1}, \quad 0 \leq a \leq J, \quad \alpha>1, \quad \beta<0
$$

where $m(a) \equiv \operatorname{Prob}\left(a_{i} \geq a\right), a_{i}$ denoting an individual's death age. That is, (1) represents the probability of being alive at age $a$. $J$ denotes the maximum attainable age which corresponds to a 0 survival probability,

$$
m(J)=0 \quad \Leftrightarrow \quad J=\frac{-\ln \alpha}{\beta}>0 .
$$

One can obtain Yaari-Blanchard's perpetual youth model with a survival probability $m(a)=e^{-\beta a}$ for $\alpha=0$ and $\beta>0$ as a limiting case [Blanchard (1985)]. Population in this economy is stable in that, in the absence of migratory movements, the age distributions of fertility and mortality rates have remained constant for a long enough time. Therefore, the age distribution and the growth rate of population are constant as well [Schoen (1988)]. We do not consider explicitly the age distribution of the fertility rate in our model. However, we will show the links between parameters $\alpha$ and $\beta$, the population growth rate and the average fertility rate later on [see equation (9)]. Increments in both $\alpha$ and in $\beta$ imply higher survival probabilities, but in a different manner. An increase in $\alpha$ means a reduction in young individuals' mortality; however, a higher $\beta$ is equivalent to lower mortality for old individuals.

The $p d f$ associated with the distribution function $\operatorname{Prob}\left(a_{i} \leq a\right) \equiv 1-m(a)$ as $p(a)$

$$
p(a)=\frac{d[1-m(a)]}{d a}=\frac{-\beta e^{-\beta a}}{\alpha-1}>0 .
$$

\footnotetext{
${ }^{3}$ This Section closely follows the first Section in Boucekkine et al. (2002).
} 
In words, (3) represents the probability of dying at the age a. Denoting by $x$ a random variable meaning "remaining lifetime until death", one obtains the probability of dying at the age $x+a$ for an individual of age $a$ from (1) and (3) as

$$
p(x / a)=\frac{p(x+a)}{m(a)}, \quad 0 \leq x \leq J-a .
$$

From (4) one obtains the (conditional) life expectancy for an individual of age a, or the average number of additional periods that one individual of age $a$ expects to live as

$$
\begin{aligned}
E V(a) & =E[x / a]=\int_{0}^{J-a} x p(x / a) d x= \\
& =\frac{e^{-\beta a}\left\{1-[1+(J-a) \beta] e^{-\beta(J-a)}\right\}}{\beta\left(e^{-\beta a}-\alpha\right)} .
\end{aligned}
$$

From (5) and (2) it is possible to solve $E V(0)$ for two particular cases: $i$ ) the life expectancy at age $a=J, E V(J)=0$; and $i i)$ at age $a=0$, i.e., the life expectancy at birth (to which we will refer as, simply, life expectancy),

$$
E V(0)=\frac{1}{\beta}-\frac{\alpha \ln \alpha}{(\alpha-1) \beta}>0
$$

One can obtain, also as a limiting case for $\alpha=0$ and $\beta>0$, Yaari-Blanchard's perpetual youth model [Blanchard (1985)] with a life expectancy which turns out to be age independent, $E V(a)=1 / \beta$.

$E V(0)$ is increasing both in $\beta$ and in $\alpha$, but (as we mentioned above) increases in $\alpha$ and in $\beta$ give rise to different kinds of increments in life expectancy. Along these lines, Kalemli-Ozcan (2002b), p. 411, claims that during the last two centuries life expectancy at birth has doubled in most parts of the world mostly due to larger falls in child rather than adult mortality. Boucekkine et al. (2002), quoting Kelley and Schmidt (1995), claim that in less developed countries mortality drops concentrate on young and working age individuals. Zhang, Zhang and Lee (2001) claim that 
during the early stages of mortality falls these concentrate on the younger population (at ages previous to reproduction); but as mortality keeps going down from low enough levels, most additional years are gained at ages after retirement age. From (6) it is easy to show that ${ }^{4}$

$$
\frac{\partial E V(0)}{\partial \beta}=-\frac{1}{\beta} E V(0)>0, \quad \frac{\partial E V(0)}{\partial \alpha}=\frac{-(\alpha-1-\ln \alpha)}{\beta(\alpha-1)^{2}}>0 .
$$

Population is assumed to grow at an exogenous, constant rate $n$, so that the measure of births at $\tau$ can be expressed as

$$
\pi(\tau)=\zeta e^{n \tau}, \quad \zeta>0, \quad n \geq 0
$$

Given (1), (2) and (7), after some algebra, one obtains the measure of population at $\tau$ as the sum of measures of individuals born between $\tau$ and $\tau-J$ who have survived until $\tau$,

$$
P(\tau)=\int_{\tau-J}^{\tau} \pi(t) m(\tau-t) d t=\zeta e^{n \tau} \kappa,
$$

where $\kappa$ is defined as

$$
\kappa=\frac{\alpha \beta\left(1-\alpha^{n / \beta}\right)+n(\alpha-1)}{(\alpha-1)(\beta+n) n}>0 .
$$

From (7) and (8) it is easy to interpret $\kappa$ as the inverse of the fertility ratio, where this is defined as the ratio of the measure of births to the measure of total population at (any) moment $\tau, 1 / \kappa=\pi(\tau) / P(\tau)$. Thus, an increase in life expectancy (regardless of whether $\alpha$ or $\beta$ becomes larger) implies two extreme cases: $i$ ) a higher population growth rate $n$ if the fertility ratio is kept constant, or $i i$ ) a reduction in the fertility ratio $\kappa^{-1}$ if the population growth rate is unchanged. ${ }^{5}$

The demographic structure that we use here, characterized by (1) and $n$ [or, alternatively, by $(1)$ and $\kappa]$ is exogenous because neither the number of children

\footnotetext{
${ }^{4}$ The sign of $\partial E V(0) / \partial \alpha$ is proven by defining $f(\alpha)=\alpha-1-\ln \alpha$, and checking that $f(1)=0$, $f^{\prime}(1)=0$ and $f^{\prime \prime}(\alpha)=\alpha^{-2}>0$. Recall the restriction $\alpha>1$.

${ }^{5}$ See Kalemli-Ozcan (2002b) who reports the fall in fertility rates for Holland, Sweden, United Kingdom, Africa, Asia and Latin America.
} 
is an individual choice, nor the survival probability depends, for instance, on per capita income or (private or public) health expenditure. ${ }^{6}$

From (1), (7) and (8) it is possible to obtain the probability density function for age $a$ as the ratio of the measure of individuals born at $\tau-a$ and still alive at $\tau$ to total population at $\tau$

$$
f(a)=\frac{\pi(\tau-a) m(a)}{P(\tau)}=\frac{e^{-n a}\left(e^{-\beta a}-\alpha\right)}{(1-\alpha) \kappa} .
$$

The instantaneous mortality rate at age a, denoted as $\xi(a)$, is defined as the ratio of the measure of individuals who die at age $a$ to the measure of individuals surviving until the age $a$. Thus, from (1), (3) and (7) one obtains

$$
\xi(a)=\frac{-d m(a) / d a}{m(a)}=\frac{p(a)}{m(a)}=\frac{-\beta e^{-\beta a}}{\alpha-e^{-\beta a}}>0,
$$

which is strictly increasing in $a$ (recall that we are assuming that $\alpha>1$ and $\beta<0$ ):

$$
\frac{\partial \xi(a)}{\partial a}=\frac{\alpha \beta^{2} e^{-\beta a}}{\left(\alpha-e^{-\beta a}\right)^{2}}>0
$$

Observed mortality rates are not strictly increasing with age, however, because they fall during childhood. ${ }^{7}$ Once more, we can obtain Yaari-Blanchard's perpetual youth model with a positive, age independent mortality rate as a limiting case: if $\alpha=0$ and $\beta>0$, then $\xi(a)=\beta>0$. [See Blanchard (1985).] The simplicity of the demographic structure that we are using here [as only three parameters $\alpha, \beta$ and $n$, and two equations, (1) and (9), are needed if migratory movements are absent], make this demographic structure highly attractive for theoretical models.

\footnotetext{
${ }^{6}$ See, among others, Kalemli-Ozcan $(2002 \mathrm{~b})$ as an example of endogenous demographic structure, or Zhang and Zhang (2003) and references therein on effects of social security on fertility rates.

${ }^{7}$ For the U.S. case, for instance, at least since the 1940's the death rate attains its minimum at the 5-14 year age group, regardless of sex and race. See Vital Statistics, in U.S. Census Bureau, Statistical Abstract of the United States: 2004-2005, Table 96, p. 75.
} 
The mean age and the median age of population, $\bar{a}$ and $\hat{a}$, respectively, can be obtained from (2) and (10) as $\bar{a}=\int_{0}^{J} a f(a) d a$ and $0.5=\int_{0}^{\hat{a}} f(a) d a$, or

$$
\bar{a}=\frac{1}{(\alpha-1) \kappa}\left\{\frac{\alpha\left[1-(1+n J) e^{-n J}\right]}{n^{2}}-\frac{1-[J(\beta+n)+1] e^{-J(\beta+n)}}{(\beta+n)^{2}}\right\},
$$

and

$$
0.5=\frac{\alpha(\beta+n)-n}{(\alpha-1) \kappa(\beta+n) n}+\frac{1}{(\alpha-1) \kappa}\left[\frac{e^{-(\beta+n) \hat{a}}}{\beta+n}-\frac{\alpha}{n} e^{-n \hat{a}}\right] .
$$

In spite of the simplicity of this demographic model, it is possible to approximate observed age distributions in terms of life expectancy, maximum age and median age reasonably well. [See Table 1]

\section{[INSERT TABLE 1 AROUND HERE]}

The pattern is clear: falls in the rate of population growth and in the rate of mortality accompany increments in life expectancy at birth and in the mean age, median age and maximum attainable age.

For illustration purposes only, Figures 1.a - bhow how changes in $\alpha$ affect the growth rate $n$, the life expectancy $E V(0)$, the mean age $\bar{a}$ and the median age $\hat{a}$. Falls in the mortality rate through increments in $\alpha$ force population aging: life expectancy, mean age and median age are raised; likewise, the population growth rate $n$ increases. Although not shown for reasons of space, differences about whether the origin is an increase in $\alpha$ or in $\beta$ (or whether $n$ or $\kappa$ is kept constant) are purely quantitative.

\section{[INSERT FIGURES 1.A-B AROUND HERE]}

\section{The economy}

Following Boucekkine et al. (2002), time is represented as a continuous variable. At each instant $\tau$ there exists a continuum of cohorts born at different instants $t$. 
A unique good is produced and it can be consumed, but not accumulated in the form of physical capital. Its price is normalized to one. Production technology uses human capital as the only production factor.

We assume that perfect annuity markets exist. Individuals do not save in physical capital (it does not exist), but in annuities. This kind of asset yields a return to its holder as long as he/she is alive. After his/her death, the property of the asset goes back to the insurance company that issued the asset. Thus, even if there were physical capital and individuals were not altruistic (so that they did not intend to leave bequests to their heirs), they would always prefer to save in annuities rather than in physical capital. The return of annuities would always be higher than that of physical capital because, in exchange, they would give back the annuities to the issuing company in case of death. This way the problem posed by unintended positive bequests is removed. Assuming that negative bequests are forbidden by law, individuals would also prefer to borrow in annuities. In exchange for cancelling out the debt in case of death, borrowers are forced to paying an extra return that compensates the lending company for the default risk in case of death. ${ }^{8}$

\subsection{The individual problem}

Denoting by $t$ birth date and $\tau$ calendar time, the problem that an individual faces consists in finding the consumption path $C(t, \tau)$, the length of the education period $T(t)$ and the retirement age $R(t)$ which maximize his/her expected lifetime utility. ${ }^{9}$

\footnotetext{
${ }^{8}$ This type of institution is often used in theoretical models as a means to avoid accidental bequests. For instance, Zhang, Zhang and Lee (2001), Fuster (1999), De la Croix and Licandro (1999), Boucekkine et al. (2002). In the following section we show that, first, the instantaneous return of annuities depends on the individual's age. Regardless of whether he/she is a borrower or a lender, the return is equal to the instantaneous probability of death which depends on age as we have seen in (11). And, second, insurance companies issuing annuities obtain zero profits in equilibrium. [Yaari (1965).]

${ }^{9}$ In our case retirement does not obey workers' health related issues, for instance, but leisure time preference. [See Sabatini and Mitchell (1999).]
} 
Workers' education has both micro consequences (higher labor income) and, as we will see later on, macro consequences (eventually, higher economic growth for the whole economy in the aggregate).

Instantaneous utility depends linearly on consumption. ${ }^{10}$ Disutility from time spent on education and working (i.e., other than in retirement) goes up as individual's age $\tau-t$ increases. It also depends negatively on average human capital in the economy $\bar{H}(t)$ at birth. Given that we will consider only steady state paths along which individual choices $R(t)$ and $T(t)$ remain time invariant, the marginal disutility of postponing retirement an additional period must be proportional to $\bar{H}(t)$. This is so because marginal utility out of the additional labor income obtained as a result of postponing retirement age one period is also proportional to $\bar{H}(t)$. In sum, if devoting time to education and working means less leisure time, lifetime utility depends negatively on retirement age.

Thus, expected lifetime utility is given by

$$
\int_{t}^{t+J} C(t, \tau) m(\tau-t) d \tau-\frac{\bar{H}(t)}{\phi} \int_{t}^{t+R(t)}(\tau-t) m(\tau-t) d \tau, \quad \phi>0
$$

where $1 / \phi$ stands for the disutility which both education and work time represent in terms of lost leisure.

The human capital with which this individual enters the labor market $h(t)$ depends on the number of periods devoted to education $T(t)$ and on the human capital in the economy at the time of his/her birth $\bar{H}(t)$. In particular,

$$
h(t)=\mu \bar{H}(t) T(t), \quad \mu>0 .
$$

There is, therefore, an externality in the production of human capital. It seems reasonable to assume that for a given education period, the human capital the

\footnotetext{
${ }^{10}$ We first tried to use a $C R R A$ (logarithmic, in particular) instantaneous utility function in order to obtain an explicit solution for consumption in a previous version of this article. However, the non linearity of the model increased substantially, giving rise to a multiplicity of solutions.
} 
individual accumulates is higher the higher the knowledge in the economy as a whole. This is, therefore, a public good that individuals enjoy but do not have to pay for. Similar mechanisms have been used previously in the theoretical literature. [See, e.g., Zhang and Zhang (2003), Zhang, Zhang and Lee (2001)-(2003), Azariadis and Drazen (1990), Lucas (1988), Lucas (1990), Einarsson and Marquis (1996), Echevarría (2003)-(2004) or Nerlove et al. (1993)]. Also, empirical evidence largely supports the positive effect of class and school composition on individual students' educational attainment or the positive effect of local workers with longer education upon individual wages. [See Benabou (1993) and references therein.]

During his/her active life the individual is paid a gross wage per unit of efficient labor equal to $\omega(\tau)$, and pays a (pay-as-you-go) social security tax at a constant rate $s \in(0,1)$. Thus, the net labor income obtained by this individual at time $\tau$ is equal to

$$
w(t, \tau)=(1-s) \omega(\tau) h(t)
$$

For simplicity, we assume that there is no depreciation of individual human capital while individuals remain on-the-job. Along these lines, Stokey and Rebelo (1995) claim that the largest source of depreciation of aggregate human capital comes from the fact that lifetimes are finite. Therefore, $O L G$ models allow a more satisfactory treatment of this issue than infinite horizon representative agent models. This, in turn, raises a new problem: how human capital is transmitted from one generation to the next. In our model current generations learn from previous generations: they take advantage of the accumulated knowledge in the society when they are in their education period.

After retirement, the individual is paid a pension benefit equal to $b(t)$. The relationship between social security contribution and pension benefit is given by the replacement rate $\vartheta$ which we define (purely for analytical convenience) in terms of 
average net wage income obtained during the active period,

$$
b(t)=\vartheta(1-s) \bar{w}(t),
$$

where

$$
\bar{w}(t) \equiv\left\{\begin{array}{l}
\int_{t+T(t)}^{t+R(t)} \frac{h(t) \omega(\tau)}{R(t)-T(t)} d \tau=h(t) \bar{\omega}(t), \quad \text { if } R(t)>T(t) \\
0, \quad \text { if } R(t)=T(t)
\end{array}\right.
$$

denotes the average gross wage income earned along the same period. That is, $\bar{\omega}(t)$ $\equiv[R(t)-T(t)]^{-1} \int_{t+T(t)}^{t+R(t)} \omega(\tau) d \tau$ represents the average gross wage per efficiency unit earned while active. ${ }^{11,12}$ This way, when making his/her optimal plan for education, the individual takes into account that more education time not only means higher wages while active, but also higher pension benefits while retired.

In short, the problem that the individual born at time $t$ faces can be formally expressed as

$$
\max _{\{C(t, \tau)\}_{\tau=J}^{\tau=J}, T(t), R(t)} \int_{t}^{t+J} C(t, \tau) m(\tau-t) d \tau-\frac{\bar{H}(t)}{\phi} \int_{t}^{t+R(t)}(\tau-t) m(\tau-t) d \tau
$$

subject to

$$
\left\{\begin{array}{l}
\int_{t}^{t+J} D(t, \tau) C(t, \tau) d \tau=\int_{t+T(t)}^{t+R(t)} D(t, \tau) w(t, \tau) d \tau+\int_{t+R(t)}^{t+J} D(t, \tau) b(t) d \tau \\
w(t, \tau)=(1-s) \omega(\tau) h(t) \\
h(t)=\mu \bar{H}(t) T(t) \\
b(t)=\vartheta(1-s) \bar{w}(t) \\
\bar{w}(t)=h(t) \bar{\omega}(t) \\
R(t) \leq J
\end{array}\right.
$$

where $D(t, \tau)$ denotes the discount factor that applies between $t$ and $\tau$; that is, the price that an individual pays in $t$ for one unit of consumption at time $\tau$ (contingent on being alive at that time).

\footnotetext{
${ }^{11}$ In some countries pension benefits are linked to the worker's wage history: that is the case, among others, of the US [Diamond and Gruber (1997)] and Spain [Boldrin et al. (1997)]. In other countries, such as the UK, Holland or Sweden, pension benefits are the universal type. [See Miles (1999).] Zhang and Zhang (2003) assume a mixed setup: part of the pension benefits is based on the wage income obtained during the active period, while the rest is of a universal nature.

${ }^{12}$ Pension benefits in our model are proportional to the average wage income earned while active for simplicity, but alternative assumptions could be made. For instance, the relationship between pension benefits and average wage income in the US and in Spain is increasing, of course, but concave. This might increase the incentives to early retirement. [See Diamond and Gruber (1997), pp. 7 y 8, and Jiménez-Martín and Sánchez (1999) pp. 49 and 50].
} 
Upon substituting the second to fourth restrictions into the first one in (20), one obtains the following Lagrangian

$$
\begin{aligned}
\mathcal{L} & =\int_{t}^{t+J} C(t, \tau) m(\tau-t) d \tau-\frac{\bar{H}(t)}{\phi} \int_{t}^{t+R(t)}(\tau-t) m(\tau-t) d \tau \\
& -\lambda(t)\left\{\int_{t}^{t+J} D(t, \tau) C(t, \tau) d \tau-\int_{t+T(t)}^{t+R(t)} D(t, \tau)(1-s) \omega(\tau) \mu \bar{H}(t) T(t) d \tau\right. \\
& \left.-\int_{t+R(t)}^{t+J} D(t, \tau) \vartheta(1-s) \bar{\omega}(t) \mu \bar{H}(t) T(t) d \tau\right\}-v(t)[R(t)-J]
\end{aligned}
$$

$\lambda(t) \geq 0$ denotes the Lagrange multiplier associated with the intertemporal budget constraint (the marginal utility of income), and $v(t) \geq 0$ is the Lagrange multiplier associated with the restriction that retirement age cannot exceed the maximum age limit $J$. Notice that $(24)$ is linear in $C(t, \tau)$, so that we are implicitly assuming that consumption is non-negative.

In short, the problem that the individual born at time $t$ faces can be formally expressed as

$$
\max _{\{c(t, \tau)\}_{\tau=t}^{\tau=J}, T(t), R(t)} \int_{t}^{t+J} c(t, \tau) m(\tau-t) d \tau-\frac{\bar{H}(t)}{\phi} \int_{t}^{t+R(t)}(\tau-t) m(\tau-t) d \tau
$$

subject to

$$
\left\{\begin{array}{l}
\int_{t}^{t+J} D(t, \tau) c(t, \tau) d \tau=\int_{t+T(t)}^{t+R(t)} D(t, \tau) w(t, \tau) d \tau+\int_{t+R(t)}^{t+J} D(t, \tau) b(t) d \tau \\
w(t, \tau)=(1-s) \omega(\tau) h(t) \\
h(t)=\mu \bar{H}(t) T(t) \\
b(t)=\vartheta(1-s) \bar{w}(t) \\
\bar{w}(t)=h(t) \bar{\omega}(t) \\
\bar{\omega}(t) \equiv[R(t)-T(t)]^{-1} \int_{t+T(t)}^{t+R(t)} \omega(\tau) d \tau \\
R(t) \leq J
\end{array}\right.
$$

where $D(t, \tau)$ denotes the discount factor that applies between $t$ and $\tau$; i.e., the price that an individual pays in $t$ for one unit of consumption at time $\tau$ (contingent on being alive at that time).

Upon substituting the second to fourth restrictions into the first one in (20), one 
obtains the following Lagrangian

$$
\begin{aligned}
\mathcal{L} & =\int_{t}^{t+J} c(t, \tau) m(\tau-t) d \tau-\frac{\bar{H}(t)}{\phi} \int_{t}^{t+R(t)}(\tau-t) m(\tau-t) d \tau \\
& -\lambda(t)\left\{\int_{t}^{t+J} D(t, \tau) c(t, \tau) d \tau-\int_{t+T(t)}^{t+R(t)} D(t, \tau)(1-s) \omega(\tau) \mu \bar{H}(t) T(t) d \tau\right. \\
& \left.-\int_{t+R(t)}^{t+J} D(t, \tau) \vartheta(1-s) \bar{\omega}(t) \mu \bar{H}(t) T(t) d \tau\right\}-v(t)[R(t)-J],
\end{aligned}
$$

where $\bar{\omega}(t) \equiv[R(t)-T(t)]^{-1} \int_{t+T(t)}^{t+R(t)} \omega(\tau) d \tau, \lambda(t) \geq 0$ denotes the Lagrange multiplier associated with the intertemporal budget constraint (the marginal utility of income), and $v(t) \geq 0$ is the Lagrange multiplier associated with the restriction that retirement age cannot exceed the maximum age limit $J$. Notice that $(24)$ is linear in $c(t, \tau)$, so that we are implicitly assuming that consumption is non-negative.

The corresponding first order necessary conditions are given by

$$
\begin{gathered}
\frac{\partial \mathcal{L}}{\partial c(t, \tau)}=0 \quad \Leftrightarrow m(\tau-t)=\lambda(t) D(t, \tau), \\
\frac{\partial \mathcal{L}}{\partial R(t)}=0 \Leftrightarrow \frac{\bar{H}(t)}{\phi} R(t) m[R(t)]= \\
\lambda(t) \mu \bar{H}(t) T(t) \omega[t+R(t)](1-s) D[t+R(t), t] \\
-\lambda(t) \vartheta(1-s) \bar{\omega}(t) \mu \bar{H}(t) T(t) D[t+R(t), t] \\
+\lambda(t) \int_{t+R(t)}^{t+J} D(t, \tau) \vartheta(1-s) \mu \bar{H}(t) T(t) \frac{\partial \bar{\omega}(t)}{\partial R(t)} d \tau-v(t),
\end{gathered}
$$

where

$$
\begin{gathered}
\frac{\partial \bar{\omega}(t)}{\partial R(t)}=\frac{\omega[t+R(t)] \times[R(t)-T(t)]-\int_{t+T(t)}^{t+R(t)} \omega(\tau) d \tau}{[R(t)-T(t)]^{2}}, \\
R(t) \leq J, \quad v(t)[R(t)-J]=0, \quad v(t) \geq 0
\end{gathered}
$$




$$
\begin{aligned}
\frac{\partial \mathcal{L}}{\partial T(t)}= & 0 \Leftrightarrow 0=\lambda(t) \int_{t+T(t)}^{t+R(t)} D(t, \tau)(1-s) \omega(\tau) \mu \bar{H}(t) d \tau \\
- & \lambda(t) \mu \bar{H}(t) T(t) \omega[t+T(t)](1-s) D[t+T(t), t] \\
& +\lambda(t) \int_{t+R(t)}^{t+J} \vartheta(1-s) \bar{\omega}(t) \mu \bar{H}(t) D(t, \tau) d \tau \\
+ & \lambda(t) \int_{t+R(t)}^{t+J} D(t, \tau) \vartheta(1-s) \mu \bar{H}(t) T(t) \frac{\partial \bar{\omega}(t)}{\partial R(t)} d \tau
\end{aligned}
$$

where

$$
\frac{\partial \bar{\omega}(t)}{\partial T(t)}=\frac{-\omega[t+T(t)] \times[R(t)-T(t)]+\int_{t+T(t)}^{t+R(t)} \omega(\tau) d \tau}{[R(t)-T(t)]^{2}} .
$$

In (26) we obtain that the marginal disutility of postponing retirement age for one additional period (in terms of lost leisure) must be equal to the marginal utility out of the augmented consumption that the additional income allows. Suppose that the retirement age is postponed one additional period. Note that $i) \lambda(t)$ represents the expected marginal utility out of income; $i i)$ the sum of the terms that multiply $\lambda(t)$ is the marginal increase of the discounted future labor income; and $i i i) v(t)$ is the expected marginal utility out of increasing the maximum lifetime horizon $J$ (relevant if the restriction $R(t) \leq J$ is binding).

By definition, one has that $D(t, t) \equiv 1$ and that $m(t-t)=m(0)=1$; therefore, from (25) we obtain

$$
\lambda(t)=1, \quad \text { and } \quad m(\tau-t)=D(t, \tau)
$$

Given that the utility function is linear in $c(t, \tau)$, so is the Lagrangian (24): if no restrictions are imposed on the optimal consumption plan, the maximum of (24) is not well defined unless $m(\tau-t)=D(t, \tau)$. That is, budget constraint and indifference curve coincide. If one imposed the non-negativity of $c(t, \tau)$ in an explicit manner, then (24) should be rewritten allowing for slackness variables. In that case, the equality between the discount factor $D(t, \tau)$ and the survival probability $m(\tau-t)$ would be obtained only for interior solutions. 
Denoting the instantaneous return of an annuity by $r(x)$, from (1) and (31) one obtains $\exp \left(-\int_{t}^{\tau} r(x) d x\right)=\left[\alpha-e^{-\beta(\tau-t)}\right](\alpha-1)^{-1}$. Upon differentiating both sides with respect to $\tau$, we obtain that

$$
r(\tau)=\frac{-\beta e^{-\beta(\tau-t)}}{\alpha-e^{-\beta(\tau-t)}} \equiv \xi(\tau-t)
$$

In other words, the instantaneous rate of return at time $\tau$ for an individual born at $t$ is identical to his/her instantaneous mortality rate defined in (11). An implication of (32) is that, assuming that insurance companies issuing annuities are risk neutral and perfectly competitive, they obtain zero profits (as expected). Denoting the stock of assets at time $\tau$ of an individual born at $t$ by $W(t, \tau)$, the costs of the insurance company would be equal to $r(\tau) W(t, \tau)$. But its revenues would be equal to $\xi(\tau-t) W(t, \tau)$ because a fraction $\xi(\tau-t)$ of individuals of age $\tau-t$ would give back all their assets to the company on dying.

As we will see in subsection 3.2, wages per efficiency unit $\omega$ are constant, so that the derivatives in (27) and (30) are identically equal to zero, and $\bar{\omega}(t)=\omega$. Therefore, from (25) and (31) we obtain that (26) can be rewritten as

$$
\begin{gathered}
-\frac{\bar{H}(t)}{\phi} R(t) m[R(t)]+\mu \bar{H}(t) T(t) \omega(1-s) m[R(t)] \\
-\vartheta(1-s) \omega \mu \bar{H}(t) T(t) m[R(t)]-v(t)=0 .
\end{gathered}
$$

There are two open possibilities. $i$ ) If the optimal $R(t)$ is an interior solution, $R(t)<J$, then $v(t)=0$. From $(33)$ one obtains $R(t)=\phi \mu T(t)(1-s)(1-\vartheta) \omega$. ii $)$ If the optimal $R(t)$ is a corner solution, then $v(t) \geq 0$ and $R(t)=J$. Thus, we will have that

$$
R(t)=\min \{\phi \mu T(t)(1-s)(1-\vartheta) \omega, J\}
$$

For the same reason, given (31) and (34), (29) can be rewritten as 


$$
\begin{aligned}
T(t) m[T(t)]= & \int_{t+T(t)}^{t+\min \{\phi \mu T(t)(1-s)(1-\vartheta) \omega, J\}} m(\tau-t) d \tau \\
& +\int_{t+\min \{\phi \mu T(t)(1-s)(1-\vartheta) \omega, J\}}^{t+J} \vartheta m(\tau-t) d \tau .
\end{aligned}
$$

Notice that (35) implies that $T(t)=T$ and, therefore, from (34) we have that $R(t)=R$. That is, optimal education time length and retirement age are constant. A key parameter for our discussion and one we will use repeatedly is $\eta \equiv \phi \mu$. If we define $\eta_{0} \equiv 1 /[(1-s)(1-\vartheta) \omega]$, the following two equations characterize $T$ and $R$

$$
R=\min \left\{\frac{\eta}{\eta_{0}} T, J\right\}
$$

and

$$
T m(T)=\int_{T}^{\min \left\{\frac{\eta}{\eta_{0}} T, J\right\}} m(\tau) d \tau+\int_{\min \left\{\frac{\eta}{\eta_{0}} T, J\right\}}^{J} \vartheta m(\tau) d \tau
$$

\subsection{Technology of aggregate production}

We assume that production technology is linear in human capital,

$$
Y(\tau)=\omega H(\tau), \quad \omega>0
$$

where $Y(\tau)$ denotes aggregate production and $H(\tau)$ aggregate human capital at $\tau$. The latter is equal to the sum of individual stocks of human capital across workers of different ages (born at different $t$ 's, but active at $\tau$ ). This is, in sum, a vintage model as explained in detail in subsection 3.4. Therefore not only is the time $\tau$ at which human capital is measured relevant, but so is the education length $T$, retirement age $R$ and age distribution of the population. Marginal productivity of human capital $\omega$ is constant and equals the (gross) wage per unit of efficiency. The parallelism with $A K$ technologies in which production is proportional to the

stock of aggregate capital in equilibrium is obvious. [See, among others, Barro and Sala-i-Martín (1995) for details.] 


\subsection{School and retirement in equilibrium}

In this subsection we characterize optimal education periods and retirement age. We will make the following distinction:

a) interior solutions: $0<T<R<J$, and

b) corner solutions which, in turn, can be of two types:

b.1) $0<T<R=J$. In this case, planned retirement is given by the maximum lifetime horizon, and education period is equal to the upper bound for interior solutions for $T$ (which we will characterize later on); and

b.2) $0=T=R<J$. In this case individuals choose neither to invest in human capital nor to enter the labor market. If so, both labor income and pension benefits are zero. This is possible given our assumption of linear utility from consumption.

\subsubsection{Interior solution: $0<T<R<J$.}

In this subsection we find the conditions upon parameter $\eta$ for the existence and uniqueness of an interior solution. If the solution is interior, from (36) we obtain that

$$
R=\frac{\eta}{\eta_{0}} T
$$

where a necessary condition that $\eta$ and $\eta_{0}$ (or, equivalently, $\eta, s, \vartheta$ and $\omega$ ) must satisfy for $R>T>0$ is that

$$
\eta>\eta_{0} .
$$

Intuitively, given the definitions of $\eta$ and $\eta_{0}$, for individuals to devote a part of their lifetimes to education and a part to active work one needs: $i$ ) high gross wages (high $\omega$ ), ii) low social security contribution rates (low s), iii) high productivity of investment in human capital (high $\mu$ ), iv) low disutility of time not devoted to leisure (high $\phi$ ), and $v$ ) low replacement rates (low $\vartheta$ ). 
Note that if the solution is interior, $R$ is proportional to $T$ and, in particular, $R=\eta(1-\vartheta)(1-s) \omega T$. Therefore, for a given $\vartheta$, the discouraging effect that a higher $s$ has upon $T$ is enlarged when we look at the effect upon $R$. This point will be relevant when we carry out our numerical exercise en Section 4.

Assuming that $\eta>\eta_{0}$, from (36), (37) and (39), optimal $T$ is given by

$$
\operatorname{Tm}(T)=G_{1}(T)+G_{2}(T)
$$

where we have defined

$$
G_{1}(T) \equiv \int_{T}^{\frac{\eta}{\eta_{0}} T} m(\tau) d \tau, \quad \text { and } \quad G_{2}(T) \equiv \vartheta \int_{\frac{\eta}{\eta_{0}} T}^{J} m(\tau) d \tau .
$$

In other words, for $T$ to be optimal the cost of an additional education period (in terms of foregone wages) must be equal to the increment in the sum of expected discounted future wages plus expected pension benefits as a result of that additional learning period.

Besides, following the case of interior solution so that $R<J$, (39) implies that

$$
T<\frac{J \eta_{0}}{\eta} \equiv T_{\max }(\eta)<J
$$

There is, therefore, an upper bound for the optimal $T$ (not only for interior solutions, but -as we will see- also for corner solutions in which $R=J$ and $T \equiv T_{\max }$ ).

Introducing unfunded social security with positive $\vartheta$ and $s$, raises the lower bound of $\eta$ for interior solutions slightly higher than the one required in Boucekkine et al. (2002). [See Boucekkine et al. (2002), Lemma 2.3, p. 350, who obtain $\eta>2$ as a necessary condition for interior solution]. Assuming [as Boucekkine et al. (2002)] $\omega=1$, one has that $\eta_{0}=[(1-s)(1-\vartheta)]^{-1}$ need not be higher than 2. For instance, the productivity of education time in the production of individual human capital need not be so high as to induce individuals to spend a fraction of their lifetimes on accumulating knowledge. Why? Because in the presence of a social security system 
like ours (unfunded and whose pension benefits depend positively on earned labor income in the past) pension benefits represent an additional incentive to the wage income obtained during the active period. In other words, pension benefits reduce the depreciation of human capital that a finite active period represents for workers.

A graphical argument may be useful to understand the last discussion. [See Figure 2.]

\section{[INSERT FIGURE 2 AROUND HERE]}

The area $\operatorname{Tm}(T)$ must be equal to the sum of areas $G_{1}(T) \equiv \int_{T}^{\frac{\eta}{\eta_{0}} T} m(\tau) d \tau$ plus $G_{2}(T) \equiv \vartheta \int_{\frac{\eta}{\eta_{0}} T}^{J} m(\tau) d \tau$. Therefore, $G_{1}(T)$ need not be so high as when there is no social security, the optimal $T$ condition being given in that case by $\operatorname{Tm}(T)=$ $G_{1}(T) \cdot{ }^{13}$

To analyze the existence and uniqueness of the interior solution, we first define

$$
\begin{aligned}
& { }^{13} \text { If there were no social security, and given that } m(x) \text { is strictly decreasing, one would have in } \\
& \text { Figure } 2 \text { that } \\
& \qquad\left(\frac{\eta}{\eta_{0}}-1\right) T m\left(\frac{\eta T}{\eta_{0}}\right)<\underbrace{\int_{T}^{\frac{\eta \eta T}{\eta_{0}}} m(x) d x}_{G_{1}(T)}<\left(\frac{\eta}{\eta_{0}}-1\right) T m(T) . \\
& \text { If the solution for } T \text { were interior, from the second of the two previous inequalities and (41) one } \\
& \text { would get } \\
& \qquad \int_{T}^{\frac{\eta T}{\eta_{0}}} m(x) d x<\left(\frac{\eta}{\eta_{0}}-1\right)\left[\int_{T}^{\frac{\eta T}{\eta_{0}}} m(x) d x+\theta \int_{\frac{\eta T}{\eta_{0}}}^{J} m(x) d x\right] \Rightarrow \\
& \Rightarrow \eta>\left[\frac{\left.2 \int_{T}^{\frac{\eta T}{\eta_{0}}} m(x) d x+\theta \int_{\frac{\eta T}{\eta_{0}}}^{J} m(x) d x\right]}{\left.\int_{T}^{\frac{\eta T}{\eta_{0}}} m(x) d x+\theta \int_{\frac{\eta T}{\eta_{0}}}^{J} m(x) d x\right]}\right]
\end{aligned}
$$

If we assume that $\theta=0, \omega=1$ and $s=0$, we will have that $\eta>2 \eta_{0}=2$, the same condition that Boucekkine et al. (2002) obtain [See Boucekkine et al. (2002), Proposition 2.1, p. 350.] 
the following auxiliary continuous function in $x$ and $\eta$ :

$$
\begin{aligned}
M(x, \eta) \equiv & (\alpha-1)\left[x m(x)-G_{1}(x)-G_{2}(x)\right] \\
= & x\left(\alpha-e^{-\beta x}\right)+\alpha x\left[1-\frac{\eta}{\eta_{0}}\right]+\frac{e^{-\beta x}-e^{-\beta x \frac{\eta}{\eta_{0}}}}{\beta} \\
& +\vartheta \alpha\left[\frac{\eta}{\eta_{0}} x-J\right]+\vartheta\left[\frac{e^{-\beta x \frac{\eta}{\eta_{0}}}-e^{-\beta J}}{\beta}\right] .
\end{aligned}
$$

From (41), (42) and (44) one has that $T$ is an interior solution if and only if $x=T$ is a root of equation (44). Therefore, we will be able to discuss the existence and uniqueness of the interior solution upon studying the properties of $M(x, \eta)$.

Our strategy will be as follows:

i) first, we will prove that $M(x, \eta)$ is negative in the origin $x=0$ [Lemma 1] and positive at $x=T_{\max }>0$ for an interval of values of $\eta$ [Lemma 2]. The continuity of $M(x, \eta)$ will assure us that there exists at least one $x \in\left(0, T_{\max }(\eta)\right)$ for which $M(x, \eta)=0 .[$ Proposition 1$]$.

ii) second, we will show that such an $x$ is unique. [Propositions 2 and 3, respectively].

The argument is shown graphically in Figure 3.

\section{[INSERT FIGURE 3 AROUND HERE]}

The following Lemma gives us sufficient conditions for $M(x, \eta)$ to be negative at $x=0$.

Lemma 1 Assume $\beta<0$ and $\alpha>1$ : if $\vartheta>0$, then $M(0, \eta)<0$.

Proof: See Appendix.

The next step consists in obtaining the conditions on $\eta$ which guarantee that $M\left(T_{\max }(\eta), \eta\right)>0$. To this end we define the following auxiliary function $K(\eta) \equiv$ 
$M\left(T_{\max }(\eta), \eta\right)$. From $(2),(43)$ and $(44)$ it can be shown that

$$
K(\eta)=\frac{1}{\beta}\left\{\frac{\eta_{0}}{\eta}\left(\alpha^{\frac{\eta_{0}}{\eta}}-2 \alpha\right) \ln \alpha+\alpha(\ln \alpha-1)+\alpha^{\frac{\eta_{0}}{\eta}}\right\},
$$

which is continuous for $\eta>0$. We next characterize function $K(\eta)$.

Lemma 2 Assume $\beta<0, \alpha>1$ and $\eta_{0}>0$ : then i) $K(\eta)$ is continuous for $\eta>0$; ii) if $\eta=\eta_{0}$, then $K(\eta)=0$; iii) there is a unique $\eta^{*}>2 \eta_{0}$ such that $K\left(\eta^{*}\right)=0$; and iv) $K(\eta)>0$ if and only if $\eta \in\left(\eta_{0}, \eta^{*}\right)$;

Proof: See Appendix.

The function $K(\eta)$ is represented in Figure 4.

\section{[INSERT FIGURE 4 AROUND HERE]}

The following Proposition gives us the interval for $\eta$ such that there exists at least one interior solution for $T$ and $R$ satisfying $0<T<R<J, T<T_{\max }$, and equations (39) and (41).

Proposition 1 Existence. Sufficiency. Assume $\beta<0, \alpha>1, \vartheta>0$ and $\eta_{0}>0$. If $\eta_{0}<\eta<\eta^{*}$, then there is at least one interior solution for $T$ and $R$ which satisfies (39) and (41), and for which $0<T<R<J$, $T<T_{\max }$.

Proof: See Appendix.

The following Proposition gives us sufficient conditions for the uniqueness of an interior solution, i.e., for equation (44) to have a unique root.

Proposition 2 Uniqueness. Sufficiency. Assume $\beta<0, \alpha>1, \vartheta>0$ and $\eta_{0}>0$. If $\eta_{0}<\eta<\eta^{*}$, then there is a unique interior solution for $T$ and $R$ which satisfies (39) and (41), and such that $0<T<R<J$, $T<T_{\max } \cdot{ }^{14,15}$

\footnotetext{
${ }^{14}$ We owe the the last part of proof to Águeda Madoz, our research assistant.

${ }^{15}$ We believe that the proofs of Lemma 2.2 and Lemma 2.3 in Boucekkine et al. (2002) are wrong. The authors claim that "Trivially, $\lim _{x \rightarrow+\infty} M(x)=+\infty \ldots$., but this is not true. [See proof of Lemma 2.2 on page 367, and proof of Lemma 2.3 on page 368.] Notice that $m(x)$ as defined in (1) is identically equal to 0 for $x \geq J$.
} 
Proof: See Appendix.

The following Proposition gives us necessary conditions for the uniqueness of the interior solution, i.e., for equation (44) to have a unique root.

Proposition 3 Uniqueness. Necessity. Assume $\beta<0, \alpha>1, \vartheta>0$ and $\eta_{0}>0$ : if the unique solution for $T$ and $R$ is interior, that is to say, $0<T<R<J$, $T<T_{\max }$, and satisfies (39) and (41), then it must be the case that $\eta_{0}<\eta<\eta^{*}$.

Proof: See Appendix.

In the next subsection we study corner solutions.

3.3.2 Corner solutions: $0<T<R=J$ and $0=T=R<J$.

We obtain two possible corner solutions by considering four cases depending on the value of $\eta:$ i) $\left.\eta=\eta^{*}, i i\right) \eta>\eta^{*}$, iii $\eta=\eta_{0}$, and $\left.i v\right) 0<\eta<\eta_{0}$.

-Case $i): \eta=\eta^{*}$

Let us assume that $\eta=\eta^{*}$ : in this case, given (39), $T_{\max }(\eta)$ defined in (43), (41), $M(x, \eta)$ defined in (44), $K(\eta)$ in (45) and $\eta^{*}$ in Lemma $2, T=T_{\max }\left(\eta^{*}\right)$ and $R=J$ satisfy the condition for an interior solution

$$
\begin{aligned}
T_{\max }\left(\eta^{*}\right) m\left[T_{\max }\left(\eta^{*}\right)\right] & =\int_{T_{\max }\left(\eta^{*}\right)}^{J} m(\tau) d \tau+\vartheta \int_{J}^{J} m(\tau) d \tau \\
& =\int_{T_{\max }\left(\eta^{*}\right)}^{J} m(\tau) d \tau .
\end{aligned}
$$

Therefore, interior and corner solutions coincide. Notice that by definition of $\eta^{*}$, $\eta^{*}>\eta_{0}$ and, therefore, $T_{\max }\left(\eta^{*}\right) \equiv \frac{J \eta_{0}}{\eta^{*}}<J$. Moreover, $T_{\max }\left(\eta^{*}\right)$ is the unique solution which, being less than $J$, satisfies (46). The following Proposition states this result.

Proposition 4 Assume that $\beta<0, \alpha>1$ and $\eta_{0}>0$ : if $\eta=\eta^{*}$, then $T=T_{\max }\left(\eta^{*}\right)$ 
$\equiv \frac{J \eta_{0}}{\eta^{*}}$ and $R=J$ is the unique solution to (39) and (41) such that $0<T<J$.

Proof: See Appendix.

-Case $i i): \eta>\eta^{*}$

The following Proposition states the solution for optimal $T$ and $R$ for values of $\eta$ greater than $\eta^{*}$.

Proposition 5 Assume that $\beta<0, \alpha>1$ and $\eta_{0}>0$ : if $\eta>\eta^{*}$, then $T$ $=T_{\max }\left(\eta^{*}\right)=\frac{J \eta_{0}}{\eta^{*}}$ and $R=J$ is the unique solution to (36) and (37) such that $0<T<J$

Proof: See Appendix.

Note that $T=R=J$ is also a solution both in Case $i$ ) and in Case $i i$ ). However, by using an indirect utility argument, this solution is dominated by $T=T_{\max }\left(\eta^{*}\right)=$ $\frac{J \eta_{0}}{\eta^{*}}$ and $R=J$. Leisure is zero in both cases $(R=J)$ and so are pension benefits. But labor income is zero, and so is consumption, if $T=R$, while labor income and consumption are positive if $T=T_{\max }\left(\eta^{*}\right)<R$.

-Case $i i i): \eta=\eta_{0}$

Consider now the case of $\eta=\eta_{0}$. From (36) and (37) we obtain

$$
\begin{gathered}
R=\min \{T, J\}, \quad \text { and } \\
T m(T)=\int_{T}^{\min \{T, J\}} m(\tau) d \tau+\int_{\min \{T, J\}}^{J} \vartheta m(\tau) d \tau .
\end{gathered}
$$

Given that individuals never survive the age $J$, it must be the case that $T \leq J$ and, therefore, $R=T$ always. This implies, in turn, that individuals never contribute to the social security and, therefore, $\vartheta$ must be 0 if the social security budget is balanced which, in turn, must be the case along balanced growth paths. 
Thus, focusing on balanced growth paths, optimal $T$ is given by (47), (48), $T=\min \{T, J\}$ and $\vartheta=0$ so that $\operatorname{Tm}(T)=0$ which admits two solutions: $0<$ $T=R=J$, where $T=T_{\max }\left(\eta_{0}\right)$, and $0=T=R<J$. The latter is preferred to the former. Why? Consumption is zero in both cases: both labor income and pension benefits are zero because $T=R$ [recall equations (17) and (18)]. But leisure is positive $(R<J)$ in the latter case, and zero in the former $(R=J)$.

The following Proposition formalizes this result.

Proposition 6 Assume that $\alpha>1, \beta<0$ and $\eta=\eta_{0}>0$. If social security budget is balanced, then $\vartheta=0,0=T=R<J$.

Proof: See Appendix.

-Case $i v): 0<\eta<\eta_{0}$

Suppose, finally, that $0<\eta<\eta_{0}$. If this is the case, from (36) one has that i) either $R=J \leq T \eta / \eta_{0}$, so that $J \leq T \eta / \eta_{0}<T$; but this cannot be a solution, as it is meaningless: individuals would study after retirement and death; or ii) $R=T \eta / \eta_{0} \leq J$ which makes sense only if $R=T=0$. Otherwise, if $T>0$, one has that $R=T \eta / \eta_{0}<T$ and, therefore, $R<T$, which makes no sense. Individuals would retire before completing the education period and entering the labor market. The following Proposition states this result. The economic meaning of Case $i v$ ) [as that of Case $i i i)$ ] is absent: there would be no human capital, nor production, nor consumption.

Proposition 7 Assume that $\alpha>1$ and $\beta<0$. If $0<\eta<\eta_{0}$, then $0=T=R<J$. Proof: Omitted.

Table 2 summarizes the results in Propositions 2-7. Note that for $\eta=\eta_{0}$ we have only considered the possibility of social security budget balance. 


\section{[INSERT TABLE 2 AROUND HERE]}

\subsection{Aggregates}

In this subsection we obtain the aggregates for consumption and human capital at time $\tau$. To this end we weight individuals' decisions by the size of the surviving population in the living cohorts, and sum them up across birth dates.

-Aggregate consumption. From (1) and (7) aggregate consumption can be expressed as,

$$
C(\tau)=\int_{\tau-J}^{\tau} C(t, \tau) \zeta e^{n t} m(\tau-t) d t
$$

where $C(t, \tau)$ represents consumption at $\tau$ of an individual born at $t$ and $\zeta e^{n t} m(\tau-t)$ denotes the measure of population of $t$-th generation still alive at time $\tau$.

- Aggregate human capital. From (1) and (7) we obtain aggregate human capital as

$$
H(\tau)=\int_{\tau-R(\tau)}^{\tau-T(\tau)} h(t) \zeta e^{n t} m(\tau-t) d t,
$$

where the last generation to enter the labor market was born at $\tau-T(\tau)$, and the last generation to retire from their jobs was born at $\tau-R(\tau)$. The cohort born at $t$ and still in the labor force has a measure equal to $\zeta e^{n t} m(\tau-t)$, and their members have a stock of individual human capital $h(t)$, making this a vintage model.

Vintage models are often used both in economies with physical capital and in economies with human capital. In the first case the aggregate stock of capital installed in firms consists of capital goods of different ages, usually embedding different technologies (with more productive technologies in the more recent ones). In the second (i.e.,our) case, younger workers incorporate higher levels of human capital than their predecessors in a growing economy (although without their labor expertise which, for simplicity, we are not considering here). ${ }^{16}$

\footnotetext{
${ }^{16}$ Among the first type one could mention, e.g., Gittleman et al. (2003) and Jensen et al. (2001).
} 
From (50) we define average human capital at time $\tau$, first introduced in the individual human capital production function (15), as

$$
\bar{H}(\tau)=\frac{H(\tau)}{\kappa \zeta e^{n \tau}}
$$

where the denominator represents total population at time $\tau$, defined in (8).

An indicator that can be used to explain growth in vintage models is the quality of human capital. This is given by the degree of obsolescence of the human capital which, in our case, is given by the average tenure of active workers $L$, which given the age distribution (10) is equal to

$$
L(\tau)=\frac{\int_{T(\tau)}^{R(\tau)}[a-T(\tau)] e^{-n a}\left(e^{-\beta a}-\alpha\right) d a}{\int_{T(\tau)}^{R(\tau)} e^{-n a}\left(e^{-\beta a}-\alpha\right) d a} .
$$

The numerical exercises in Section 4, however, will not show a monotonic relationship between $L$ and $\gamma$.

\subsection{Social security}

Assuming that social security balances its budget on a period by period basis, the following equality must hold at each time $\tau$

$$
\int_{\tau-R(\tau)}^{\tau-T(\tau)} s \omega(\tau) h(t) \pi(t) m(\tau-t) d t=\int_{\tau-J}^{\tau-R(\tau)} b(t) \pi(t) m(\tau-t) d t .
$$

The left-hand-side represents the social security tax revenue from active generations [i.e., born after $\tau-R(\tau)$, but before $\tau-T(\tau)]$. The right-hand-side equals the pension benefits paid to retirees [i.e., individuals born after $\tau-J$, but before $\tau-R(\tau)$ ].

\subsection{Equilibrium}

Definition 1 An equilibrium path for this economy is defined as a sequence of quantities $\{T(\tau), R(\tau), C(\tau), h(\tau), H(\tau), \bar{H}(\tau), Y(\tau)\}_{\tau=0}^{\infty}$ and prices $\{\omega(\tau), D(t, \tau)=$ $m(\tau-t)\}_{\tau=0}^{\infty}$ such that

And among the second type, one could mention Boucekkine et al. (2002), Echevarría (2003)-(2004), Violante (2002), and Neuman and Weiss (1995). 
i) consumers maximize utility taking the sequences of human capital in the economy and wage per efficiency unit, and the parameters representing the social security policy $\{s, \vartheta\}$ as given;

ii) firms maximize profits taking the sequence of wage per efficiency unit as given;

iii) the government chooses the replacement rate $\vartheta$, for a given social security tax rate s such that social security budget is balanced at each instant; and

iv) goods market clears.

In this article we only consider balanced growth paths characterized by the fact that aggregate variables $\{C(\tau), H(\tau), Y(\tau)\}$ grow at a constant rate or, equivalently, per capita variables $\{h(\tau), \bar{H}(\tau), w(\tau)\}$ grow at a constant rate $\gamma$. Moreover, variables indicating education time duration $T$ and retirement age $R$ are constant and, therefore, do not depend on the worker's birth date.

\subsection{The balanced growth path}

Definition 2 Balanced growth paths in this economy are defined as sequences of quantities $\{T, R, C(\tau), h(\tau), H(\tau), \bar{H}(\tau), Y(\tau)\}_{\tau=0}^{\infty}$ and prices $\{\omega, D(t, \tau)=m(\tau-$ t) $\}_{\tau=0}^{\infty}$ such that

i) conditions i)-iv) in Definition 1 are met, and

ii) all aggregate variables in per capita terms grow at a constant rate $\gamma$.

In order to obtain the steady state growth in this economy, we first calculate the rate of growth of aggregate human capital: the sum of population growth rate plus the growth rate of average human capital. To obtain the latter we substitute (15) and (51) into (50),

$$
\bar{H}(\tau) \kappa \zeta e^{n \tau}=\int_{\tau-R}^{\tau-T} \mu \bar{H}(t) T \zeta e^{n t} m(\tau-t) d t .
$$

If we take into account that along the balanced growth path $\bar{H}(t)=\bar{H}(\tau) e^{-\gamma(\tau-t)}$ 
must hold, and we make the following change of variable $z=\tau-t$, on recalling the survival probability (1) we can rewrite the above expression as,

$$
\frac{\mu T}{\kappa} \int_{T}^{R} e^{-(\gamma+n) z}\left[\frac{\alpha-e^{-\beta z}}{\alpha-1}\right] d z=1,
$$

which implicitly characterizes the per capita growth rate $\gamma$ as a function of $\mu, T$, $\kappa, R, n, \alpha$ and $\beta$. Ceteris paribus, from (54) one can see that for higher values of retirement age $R$ or productivity in human capital production $\mu$, the economy's long run growth rate $\gamma$ will be higher. If the education time length $T$ becomes longer, then an ambiguous result shows up: $i$ ) aggregate human capital is enlarged (individual human capital stocks are higher), but $i i$ ) the share of active population becomes smaller. From (54) one has the following Proposition.

Proposition 8 Assume that $0<T<R<J$. If there is a per capita growth rate $\gamma$ that satisfies (54), it must be unique.

Proof: See Appendix.

An open question is the convergence of this economy to the steady state. Equation (50) is exactly the same as the one obtained by Boucekkine et al. (2002). [See equation (23) in Boucekkine et al. (2002), p. 353.] $]^{17}$

Given that along balanced growth paths $R(\tau)=R, T(\tau)=T, \omega(\tau)=\omega$, and $\bar{H}(t)=\bar{H}(\tau) e^{-\gamma(\tau-t)}, \pi(t)=\pi(\tau) e^{-n(\tau-t)}$, taking into account (15), (17) and (18), and after a change of variable $z=\tau-t$, the equation for social security budget balance (53) can be rewritten as

$$
s \int_{T}^{R} e^{-(\gamma+n) z} m(z) d z=\vartheta(1-s) \int_{R}^{J} e^{-(\gamma+n) z} m(z) d z .
$$

\footnotetext{
${ }^{17}$ In essence Boucekkine et al. (2002) show that the dynamics of aggregate human capital is characterized by a second order delayed differential equation with delayed derivatives. Its resolution requires numerical methods, because no mathematical result that sets conditions on the parameters ( $T$ and $R$, in particular) for it to converge is known.
} 
Finally, if the solution for $T$ and $R$ happens to be interior, the payroll tax rate $s$ and the replacement rate $\vartheta$ which balance the social security budget are unique.

Proposition 9 If $0<T<R<J$, then there is a unique pair of payroll taxreplacement rates $(s, \vartheta)$ which satisfies the social security budget balance.

Proof: See Appendix.

Thus, summing up, along the balanced growth equilibrium path conditions (36), (37), (54) and (55) must be met. This makes four non-linear equations in 4 unknowns: $T, R, \gamma$ and $\vartheta$ (for a given $s$ ). Unlike Boucekkine et al. (2002), it is not possible to obtain a relationship between $T$ and $\gamma$ in our model: $\gamma$ influences $T$ through social security budget balance which in turn affects $\vartheta$; and $T$ affects $\gamma$ through the growth rate equation. Therefore, it is not possible obtain a replica of their Proposition 3.4. [See Boucekkine et al. (2002), Proposition 3.4, p. 355.] Moreover, once the individual problem and the uniqueness of the steady state growth rate and the social security budget balance are solved analytically, all the results that follow in the next Section are strictly numerical. 


\section{A numerical example}

\subsection{Calibration}

In this Section we give values to the basic parameters to calculate numerically the steady state equilibrium for a benchmark case. In order to illustrate the role played by social security in our model, we run a first experiment in which we compare the responses of this economy to exogenous changes in life expectancy under two alternative scenarios: with and without social security. To this end, and taking some features of the U.S. economy as an example, we use two sets of parameters which give us to some approximation the same benchmark steady state. ${ }^{18}$ The summary of the two sets of parameters, the steady state theoretical values for the benchmark model and the empirically observed values are shown in Table 3. In a second experiment, we analyze the effects of changes in social security policy.

Concerning our first experiment, we choose one set of demographic parameters $\alpha, \beta$ and $n$ so that we are able to approximate the observed mean and median ages and the life expectancy at birth, $\bar{a}, \hat{a}$ and $E V$, respectively. The crude birth rate $1 / \kappa$ turns out to take a reasonable value too. ${ }^{19}$ As for the non-demographic parameters, we choose two values for each of $\mu, \phi$ and $\omega$ in such a way that the resulting $T, R$ and $\gamma$ obtained with $(s>0)$ and without $(s=0)$ social security satisfy two conditions:

\footnotetext{
${ }^{18}$ At any rate, the parameter sets must be such that $\eta_{0}<\eta<\eta^{*}$. If $\eta \leq \eta_{0}$, condition (54) is not met; and if $\eta \geq \eta^{*}$, from (55) one would have that $\vartheta$ would be infinite. Therefore, we focus on interior solutions for $T$ and $R$.

${ }^{19}$ Data on $n$ have been obtained from U.S. Census Bureau, National and Population Estimates, NST-EST2003-pop-chg, Annual Estimates of the Population Change for the United States, and Puerto Rico and State Rankings: July 1, 2002, to July 1, 2003, p.1, available at http://www.census.gov/popest/states/NST-EST2003-pop-chg.html. For EV see 2004 World Population Data Sheet of the Population Reference Bureau, Demographic Data and Estimates for the Countries and Regions of the World, p. 7, available at http://www.prb.org. For $\bar{a}$ and $\hat{a}$ see U.S. Census Bureau, National Population Estimates, Characteristics, Annual Estimates of the Population by Sex and Five Year Age Groups for the United States: April 1, 2000 to July 1, 2003 (NC-EST2003-01) p.1, available at http://www.census.gov/popest/national/asrh/NC-EST2003/ Finally, for data on $1 / \kappa$ see Vital Statistics, in U.S. Census Bureau, Statistical Abstract of the United States: 2004-2005, Table 72, p. 61.
} 
reasonably close between them and also to observed values, in particular in $R$ and $\gamma \cdot \cdot^{20}$

\section{[INSERT TABLE 3 AROUND HERE]}

Once we have set up the benchmark case, the experiment consists in generating a range of values for the life expectancy (between 40 and 100). There are 4 ways of modelling increases in life expectancy depending on, first, whether these are due to lower mortality rate for young or for old agents (higher $\alpha$ or higher $\beta$, respectively); and, second, whether the population growth rate or the crude birth rate adjusts to mortality changes (constant $n$ and lower $1 / \kappa$ or lower $n$ and constant $1 / \kappa$, respectively). Here we focus on increases in life expectancy caused by lower mortality rates for young agents and lower crude birth rates, and then we discuss how our results would differ if changes in $\beta$ or $n$ had been considered. The results are illustrated in Figures 5 and 6 . We run an additional experiment: keeping the rest of the parameters constant, we analyze the response of our theoretical economy upon changes in the social security tax rate $s$. The results are illustrated in Figure 7.

\section{$4.2 \quad$ Findings}

In our model the engine of growth is given by the change in the average human capital of the economy. Average human capital, in turn, depends on, first, individual decisions such as optimal schooling time and retirement age which (in the case of $T$ ) affect not only their own productivity, but also the share of active workers in

\footnotetext{
${ }^{20}$ The value of $s$ has been obtained from Coronado et al. (2000), p. 10. For data on $T$ see Butcher et al. (1994). Bassanini et al. (2001), p. 28, show the increasing time trend of average years of education among working population for 21 OECD countries between 1971 and 1978. As for retirement age $R$ see, e.g., Gendell (1998). For $\gamma$ see Income, Expenditures and Wealth, in U.S. Census Bureau, Statistical Abstract of the United States: 2004-2005, Table 648, p. 430. Finally, as for the replacement rate, observed values vary substantially depending on the worker's individual characteristics. See, e.g., Diamond and Gruber (1997) or Kotlikoff et al. (1999).
} 
the population as $T$ and $R$ represent entry to and exit from the labor market, respectively. And, second, demographic characteristics, namely the survival rate distribution. Thus, falls in the mortality rate (i.e., increases in life expectancy) imply both a behavior effect (through changes in $T$ and $R$ ) and a composition effect (through changes not only in the age distribution of workers, but also on the range of active ages) which in turn imply changes in the growth rate. We discuss below that both effects differ depending on whether there is an unfunded social security in the economy or not.

We graph the response of individual and aggregate variables (in Figures 5 and 6 , respectively) to increases in the life expectancy under both scenarios, i.e. with and without social security. ${ }^{21}$

As life expectancy goes up, education time length increases in both scenarios. Higher survival probabilities increase the expected flow of future wages, thereby giving incentives to extend the education time length. In eq. (37) the left-hand side represents the marginal cost of increasing $T$, and the two terms on the right-hand side represent the marginal benefit, i.e. the expected flow of future wages (pension benefits included). Without social security only the first term is present. With social security (if pensions depend on past contributions), however, an additional term shows up as the incentives to a higher $T$ increase. [See Fig. 5.a.]

Concerning the retirement age decision, we can see in equation (39) that increases in life expectancy affect $R$ through both its effect on $T$ (as in an economy without social security) and the social security policy represented by the replacement and the contribution rates $\vartheta$ and $s$, respectively (which enter the definition of $\eta_{0}$ ). Therefore, with social security the relationship between $T$ and $R$ is not proportional. In an

\footnotetext{
${ }^{21}$ The range of values for $\alpha$ goes from 3.4 to $13.5 ; n$ and $\beta$ take on the same values as in the benchmark case, 0.010 and -0.0170 , respectively. As a result, $E V$ ranges between 43.2 and 106.5, and $\kappa^{-1}$ between 0.028 and 0.015 .
} 
economy with no social security, however, increments in life expectancy give rise to increases in $R$ proportional to those in T. [See Fig. 5.c.] Our numerical experiment shows that increases in life expectancy allow a higher generosity of social security because $\vartheta$ becomes higher. Why? Workers are more productive (so that they contribute more) and their survival probabilities are higher (particularly among young agents). [See Fig. 6.a.] Therefore, (unfunded) social security gives incentives to early retirement, as it is a well known fact in the literature. This explains why as life expectancy becomes larger, the retirement age increases at a lower rate than in an economy with no social security. In fact, for high enough levels of life expectancy, $R$ is lower with than without social security. [See Fig. 5.b.] Along these lines, Fig. 5.c shows the ratio of $R$ to $T$ under both regimes: without social security it is just a constant; with social security, however, it is decreasing in $T$.

This lower increment in $R$ implies that the active life length $R-T$ increases at a lower rate with than without social security. So, even though the increment in $T$ is expected to be higher in the presence of social security (as discussed above), it is lower than what it would have been had retirement age been exogenous. [See Fig. $5 . d$.

We graph next the response of aggregate variables to increases in the life expectancy with and without social security in Figure 6. As advanced above, increases in life expectancy bring about a higher generosity of social security represented by a higher $\vartheta$. [See Fig. 6.a.]

Along a balanced-growth path economy, changes in active life parallel changes in average tenure of active workers, L. [See Fig. 6.b]. Therefore, it is not surprising that $L$ increases along with life expectancy, but at a lower rate in the presence of social security than in its absence, thus displaying a similar pattern to that one of $R-T$. This has a composition and a behavior (productivity) effect on the 
per capita growth rate of the economy. First, the share of active workers in the population falls. Why? Even though the span of active life $R-T$ becomes larger, the whole population age distribution changes: it can be shown that the weight of young individuals relatively drops, while olders' becomes relatively higher. However, in an economy without social security the proportion of active workers increases with life expectancy. This composition effect turns out to be crucial to understand the response of per capita growth as we see next. [See Fig. 6.c. $]^{22}$ Second, given the lower rate increment in average tenure, the obsolescence of active workers' human capital will increase at a lower rate too.

We finally arrive at the relationship between life expectancy and per capita growth rate, and find that it exhibits an inverted $U$ pattern under both scenarios. In the social security regime, however, the negative sloped part shows up at much lower levels of life expectancy. The explanation must be sought in the incentives to early retirement that social security introduces and that, as we have just seen, make the share of working population fall substantially. [See Fig. 6.d.]

More precisely, without social security the vintage characteristic appears to play an important role in explaining the decreasing part of the inverted $U$ : if mortality rates fall, the proportion of workers whose schooling took place a long time ago (and who have become obsolete) is higher. With social security, however, the vintage characteristic does not play such an important role: the explanation must be sought in the decrease in the share of workers: if mortality rates fall, growth rates may end up decreasing simply because workers retire earlier.

\section{[INSERT FIGURES 5 AND 6 AROUND HERE.]}

\footnotetext{
${ }^{22}$ It can be shown that in the social security case, increases in life expectancy go along increments in the dependency ratio (retirees to workers ratio), a well documented fact in real economies. [See, e.g., Diamond et al. (1997).] In the no social security case, however, increments in life expectancy accompany falls in the dependency ratio.
} 
How would our results have changed if we had allowed reductions in the population growth $n$ (instead of the crude birth rate $1 / \kappa)$ ? Or in the mortality for old agents through increments in $\beta$ (instead of the mortality for young agents through increments in $\alpha)$ ? Falls in crude birth rates along with increments life expectancy and constant population growth rate are closer to the observed demographic characteristics nowadays than constant crude birth rates and increasing population growth rates. In terms of the model, the results (not shown for space saving) change only quantitatively. Regarding whether the reduction in mortality rates affects mainly young or old individuals, Kalemli-Ozcan (2002b) claims that during the last two centuries life expectancy has doubled in most parts of the world mostly due to larger falls in child rather in adult mortality. ${ }^{23}$ It can be shown that the main difference shows up in the share of workers. If increments in $\beta$ rather than in $\alpha$ are assumed, the share of workers in an economy without social security decreases: the increase in the population size mainly takes place at older ages.

How do these results match observed facts? Or, does the existence of unfunded social security affect the response of the economy to changes in life expectancy? Let us continue with the U.S. case and split the data into two periods, 1870-1940 and 1950-2000, which can represent our two regimes: without and with social security, respectively. ${ }^{24}$ Regarding the first period, we observe what our model predicts that increased life expectancy is accompanied by three facts: $i$ ) increased average years of education, $i i)$ an inverted $U$ pattern for the average annual growth rate of per capita $G D P$, and $i i i)$ a fairly stable labor force participation. [See rows $2-4$ in

\footnotetext{
${ }^{23}$ Zhang et al. (2001) claim that during the early stages of mortality falls these concentrate on the younger population; but as mortality keeps going down from low enough levels, most additional years are gained after retirement age.

${ }^{24} 1870$ is the first year for which we have found available data for the series. Social security was first introduced by the Social Security Act of 1935, although still in 1950 (when major amendments were enacted) only about $50 \%$ of workers were covered. [See Social Security. A Brief History (available at http://www.socialsecurity.gov/history) for further details.] Additionaly, the figures for the growth rates of per capita GDP were highly affected by WWII events.
} 
Table 4. $]^{25}$ For the second period (see rows 5-7 in Table 4) the predictions of our model partly resemble observed facts: as before, augmented life expectancies go along with facts $i$ ) and $i i$ ). As for the observed aggregate (i.e., for both sexes) labor force participation, it exhibits an increase as opposed to the decline that our one-sex model predicts. Why? A major distinction between male and female labor force participation rates must be made and in particular among younger and older workers. ${ }^{26}$ Whereas participation rates among women have increased substantially, men's have declined: for those aged 16 to 24 , the decline likely reflects increases in $T$; and for the elderly, the reason may be sought in a higher availability of pensions and disability awards, i.e., social security. [See Fullerton (1999).] Our model does not predict a decline in $R$ in the social security regime, but it does predict a lower increment in the retirement age. Perhaps an extended number of observations would allow us to observe a deeper reduction in the per capita GDP growth rate and a fall in aggregate participation rates as observed among male workers.

\section{[INSERT TABLE 4 AROUND HERE]}

\section{-Social Security.}

Figure 7 shows the effects of changes in the social security contribution rate $s .{ }^{27}$ The first result is a net discouraging effect upon human capital accumulation and retirement age.

\footnotetext{
${ }^{25}$ Sources of data for the two periods differ. For the $1870-1940$ period, the following sources have been used: Maddison (1995) for education years, labor force participation and per capita GDP growth; and http://www.ac.wwu.edu/ stephan/webstuff/demographs/life.data.html for life expectancy. For the 1950-2000 period, the sources used are: Barro and Lee (2000) for education years; Vital Statistics, U.S. Census Bureau, Statistical Abstracts (several years) for life expectancy; and Fullerton (1999), Szafran (2002), U.S. Census Bureau, Statistical Abstract (2001) for labor force participation, and Penn World Table for per capita GDP.

${ }^{26}$ The difference in the observed pattern for the participation rates between men and women has been studied in the literature. See, e.g., Attanasio et al. (2004) and references therein. Two additional forces help explain the behavior of observed labor force participation rates: immigration and changes in the age distribution of population following the baby boom, neither of which our model can account for. [See Fullerton (1999).]

${ }^{27} s$ ranges between $s=0.1 \%$ and $s=40.0 \%$.
} 
A higher social security tax rate $s$ reduces the net wage $w(t)=(1-s) \omega \mu \bar{H}(t) T$ [for a given $\bar{H}(t)$ ], so that incentives to devote a fraction of lifetime to education $T$ are reduced. ${ }^{28} R$ also drops significantly when $s$ rises. As noted in subsection 3.3.1, $R=\eta(1-\vartheta)(1-s) \omega T$. Given that the replacement rate $\vartheta$ remains relatively unchanged, pension benefits fall as well, so that incentives to lengthen the education phase are reduced additionally, what generates an even larger reduction in $R$.

Given the reaction of $R$, it is easy to understand the response of the dependency ratio, $R D$ : the higher the social security contribution rate, the higher the dependency ratio, and the lower the share of active workers. This result is in line with Coile et al. (2000) and Kalemli-Ozcan (2002a), among others.

Once analyzed the behavior of $T, R$ and $R D$, the performance of $\gamma$ is as expected: higher social security contribution rates go along with lower rates of per capita growth. ${ }^{29}$

Finally, focusing on the replacement rate, $\vartheta$ remains hardly unchanged. How is it possible that (upon increasing $s$ ) $R D$ goes up and $\vartheta$ stays almost constant? Remember, first, that $\vartheta$ represents the replacement rate defined on net wages $(1-$ s)w; and, second, as noted in the beginning of this subsection, the social security budget balance condition depends on the dependency ratio, the contribution and the replacement rates and also the economy's per capita growth rate $\gamma$.

As a result, one would expect the generosity of the pension scheme to fall. One way to measure this consists in calculating the ratio of the sum of present values of expected pension benefits to the sum of present values of expected social security

\footnotetext{
${ }^{28}$ In Zhang and Zhang (2004) the effect is just the opposite, but the mechanism is different: parents pay for their children's education. Higher pay-roll taxes reduce the net wages that children will obtain and increase parents' incentives to spend more on their children's education (and have less children).

${ }^{29}$ Nevertheless, the values of $s$ for which $\gamma$ attains the maximum is not zero, but slightly positive: in our numerical example, we obtain that $\gamma=2.3 \%$ for $s=1.4 \%$. The existence of externalities in the human capital accumulation explains why positive social security may promote growth.
} 
contributions. Thus, from (1), (31), (15), (16), (17) and (18), and recalling that $\omega(\tau)=\omega$, one has

$$
G=\frac{\int_{t+R}^{t+J} D(t, \tau) b(t) d \tau}{\int_{t+T}^{t+R} D(t, \tau) s \omega h(t) d \tau}=\frac{\vartheta(1-s)\left[\alpha(J-R)+e^{-\beta J}-e^{-\beta R}\right]}{s\left[\alpha(R-T)+e^{-\beta R}-e^{-\beta T}\right]} .
$$

The relationship between $G$ and $s$ that we obtain is strictly decreasing. In fact, for low enough $s$ (in our numerical example $s<0.27$ ), the $G$ that we obtain is higher than 1 (actuarially more than fair pension benefits).

\section{[INSERT FIGURE 7 AROUND HERE]}

\section{Conclusions}

In this article we have studied the relationship between life expectancy and per capita growth rate. We have used a vintage growth model with pay-as-you-go social security where individuals choose education time and retirement age and where pensions depend on the contributions made by workers during their active lives. This way the flow of income during the retirement period also depends on the education time investment.

The results obtained in the first part of the article are analytical. We have characterized the individual's parameter space which establishes the type of solution for education length and retirement age. We have also proved the existence of, at most, one steady state per capita growth rate and of one unique steady state budget balance for the social security. In the second part we have compared the responses of the economy to exogenous changes in life expectancy under two regimes (with and without social security), obtaining numerical results.

In our model the engine of growth is given by the change in the average human capital of the economy. Average human capital depends on, first, individual decisions such as optimal schooling and retirement age which affect their own produc- 
tivity and the share of active workers in the population. And, second, demographic characteristics, namely the survival rate distribution. We have seen that increases in life expectancy imply both a behavior effect (through changes in schooling and retirement age) and a composition effect (through changes in the age distribution of workers and in the range of active workers) which in turn imply changes in the growth rate. We have found that in an economy with no social security the vintage characteristic seems to play a relevant role as the proportion of agents whose schooling was made a long time ago becomes higher with higher levels of life expectancy. However, in an economy with social security the vintage description of the economy does not play such an important role in explaining the decreasing part of the life expectancy-per capita growth locus. In this case the decrease in the share of workers as life expectancy goes up is the main factor.

Finally, we have studied the relationship between the size of the social security and per capita growth rate of the economy. We have obtained that such a relationship is mostly negative, except for very low values for the social security contribution rate. The explanation is found in the discouraging effect that social security imposes in education and, in particular, retirement age what causes a fall in the share of working population in the economy.

We believe that this line of research deserves further empirical work, especially in western economies in which life expectancy has reached substantially high levels and where there is strong debate about the sustainability of current unfunded social security systems and the convenience of postponing retirement age. 


\section{Appendix}

Proof of Lemma 1. Substituting $x=0$ into (44) one has that $M(0, \eta)=\frac{\vartheta}{\beta}$ $[1-\alpha(1-\ln \alpha)]$. Let us define $f(\alpha) \equiv 1-\alpha(1-\ln \alpha)$. If $\vartheta>0$ and $\beta<0$, $M(0, \eta)<0 \Leftrightarrow f(\alpha)>0$. Note that $f(1)=0$ and $f^{\prime}(\alpha)=\ln \alpha>0 \forall \alpha>1$. Therefore, if $\alpha>1$, then $f(\alpha)>0$ and $M(0, \eta)<0$.

Before proving Lemma 2, some preliminaries (Claims 1-8) are needed.

Claim 1. Assume $\beta<0, \alpha>1$ and $\eta_{0}>0$ : if $\eta<\eta_{0}$, then $K(\eta)$ is strictly increasing.

Proof of Claim 1. Assume that $\beta<0, \alpha>1$ and $\eta_{0}>0$. From (45) one obtains that $K^{\prime}(\eta)=\frac{\eta_{0} \ln \alpha}{\eta^{2} \beta}\left[2 \alpha-\alpha^{\frac{\eta_{0}}{\eta}}\left(2+\frac{\eta_{0}}{\eta} \ln \alpha\right)\right]=\frac{\eta_{0} \ln \alpha}{\eta^{2} \beta} g(\alpha, \eta)$, where $g(\alpha, \eta) \equiv 2 \alpha-$ $\alpha^{\frac{\eta_{0}}{\eta}}\left(2+\frac{\eta_{0}}{\eta} \ln \alpha\right)$. Given the assumptions on $\alpha, \beta$ and $\eta_{0}, K^{\prime}(\eta)>0 \Leftrightarrow g(\alpha, \eta)<0$. Thus, it is necessary to prove that if $\eta<\eta_{0}$, then $g(\alpha, \eta)<0$. Notice the following facts:

i) $g(1, \eta)=0$

ii) $\partial g(\alpha, \eta) / \partial \alpha=2-\frac{\eta_{0}}{\eta} \alpha^{\frac{\eta_{0}-\eta}{\eta}}\left(3+\frac{\eta_{0}}{\eta} \ln \alpha\right) \equiv h(\alpha, \eta)$;

iii) $h\left(\alpha, \eta_{0}\right)=-1-\ln \alpha$;

iv) $h\left(1, \eta_{0}\right)=-1$

v) $\partial h\left(\alpha, \eta_{0}\right) / \partial \alpha=-1 / \alpha<0$; therefore,

vi) $g\left(\alpha, \eta_{0}\right)<0$. We need to check how $g(\alpha, \eta)$ behaves for values of $\eta$ less than $\eta_{0}$. It is straightforward to check that:

vii) $\partial g(\alpha, \eta) / \partial \eta=\frac{\eta_{0}}{\eta^{2}} \alpha^{\frac{\eta_{0}}{\eta}}\left(3+\frac{\eta_{0}}{\eta} \ln \alpha\right) \ln \alpha>0$.

Therefore, from vi) and vii) one has that if $\eta<\eta_{0}$, then $g(\alpha, \eta)<0 \Leftrightarrow K^{\prime}(\eta)>$ 0.

Claim 2 Assume $\beta<0, \alpha>1$ and $\eta_{0}>0$. i) $K\left(\eta_{0}\right)=0$. ii) If $\eta_{0}>0$, then $K^{\prime}\left(\eta_{0}\right)>0$.

Proof of Claim 2. Assume that $\beta<0, \alpha>1$ and $\eta_{0}>0$.

i) Trivially, from $(45) K\left(\eta_{0}\right)=\frac{1}{\beta}[-\alpha \ln \alpha+\alpha(\ln \alpha-1)+\alpha]=0$.

ii) Finally, from the previous proof one has that

$K^{\prime}(\eta)=\frac{\eta_{0} \ln \alpha}{\eta^{2} \beta}\left[2 \alpha-\alpha^{\frac{\eta_{0}}{\eta}}\left(2+\frac{\eta_{0}}{\eta} \ln \alpha\right)\right]$. Evaluating $K^{\prime}(\eta)$ in $\eta=\eta_{0}$, one has $K^{\prime}\left(\eta_{0}\right)=\frac{\eta_{0} \ln \alpha}{\beta \eta_{0}^{2}}[2 \alpha-\alpha(2+\ln \alpha)]=-\frac{\alpha(\ln \alpha)^{2}}{\eta_{0} \beta}>0$.

Claim 3 If $\beta<0, \alpha>1$ and $\eta_{0}>0$, then $K(\eta)$ is negative for all $\eta<\eta_{0}$.

Proof of Claim 3. From Claim 1 and part $i$ ) in Claim 2, it is trivial. 
Claim 4 If $\beta<0, \alpha>1$ and $\eta_{0}>0$, then i) $K\left(2 \eta_{0}\right)>0$, and ii) $K^{\prime}\left(2 \eta_{0}\right)<0$.

Proof of Claim 4. Assume that $\beta<0, \alpha>1$ and $\eta_{0}>0$.

i) After some tedious algebra, it can be shown from (45) that $K\left(2 \eta_{0}\right)=\frac{\alpha^{1 / 2}}{\beta}\left(\frac{\ln \alpha}{2}+1-\alpha^{1 / 2}\right)=\frac{\alpha^{1 / 2}}{\beta} i(\alpha)$, where $i(\alpha) \equiv \frac{\ln \alpha}{2}+1-\alpha^{1 / 2}$. Given that $\beta<0, K\left(2 \eta_{0}\right)>0 \Leftrightarrow i(\alpha)<0$. Notice that, first, $i(1)=0$ and, second, $i^{\prime}(\alpha)$ $=\frac{\alpha^{-1 / 2}}{2}\left(\alpha^{-1 / 2}-1\right)<0 \Leftrightarrow \alpha>1$. Therefore, if $\alpha>1$, then $i(\alpha)<0$ and $K\left(2 \eta_{0}\right)$ $>0$.

ii) Finally, from the previous proof, one has that $K^{\prime}(\eta)=\frac{\eta_{0} \ln \alpha}{\eta^{2} \beta}\left[2 \alpha-\alpha^{\frac{\eta_{0}}{\eta}}\left(2+\frac{\eta_{0}}{\eta} \ln \alpha\right)\right]$. Evaluating $K^{\prime}(\eta)$ at $\eta=2 \eta_{0}$, one has $K^{\prime}\left(2 \eta_{0}\right)=\frac{\ln \alpha}{4 \beta \eta_{0}} j(\alpha)$, where $j(\alpha) \equiv 2 \alpha-\alpha^{\frac{1}{2}}\left(2+\frac{\ln \alpha}{2}\right)$. Given the restrictions upon $\beta$ and $\eta_{0}$, then $K^{\prime}\left(2 \eta_{0}\right)<0 \Leftrightarrow j(\alpha)>0$. Note the following facts:

ii.1) $j(1)=0$;

ii.2) $j^{\prime}(\alpha)=2-\alpha^{-1 / 2}\left(\frac{3}{2}+\frac{\ln \alpha}{4}\right)$;

ii.3) $j^{\prime}(1)=1 / 2$; and,

ii.4) $j^{\prime \prime}(\alpha)=\alpha^{-3 / 2}\left(\frac{\ln \alpha}{8}+\frac{1}{2}\right)>0$ if $\alpha \geq 1$.

Therefore, if $\alpha>1$, then $j(\alpha)>0$ and $K^{\prime}\left(2 \eta_{0}\right)<0$.

Claim 5 If $\beta<0, \alpha>1$ and $\eta_{0}>0$, then one unique $\hat{\eta} \in\left(\eta_{0}, 2 \eta_{0}\right)$ exists such that $K^{\prime}(\hat{\eta})=0$.

Proof of Claim 5. Assume that $\beta<0, \alpha>1$ and $\eta_{0}>0$. From (45) one obtains that $K^{\prime}(\eta)=\frac{\eta_{0} \ln \alpha}{\beta \eta^{2}} g(\alpha, \eta)$, where $g(\alpha, \eta) \equiv 2 \alpha-\alpha^{\frac{\eta_{0}}{\eta}}\left(2+\frac{\eta_{0}}{\eta} \ln \alpha\right)$, continuous in $\eta$ for all $\eta>0$. Therefore, $K^{\prime}(\eta)=0 \Leftrightarrow g(\alpha, \eta)=0$. Moreover, $g(\alpha, \eta)=0 \Leftrightarrow$ $l(\alpha, \eta)=m(\alpha, \eta)$, where $l(\alpha, \eta) \equiv 2 \alpha^{\frac{\eta-\eta_{0}}{\eta}}$ and $m(\alpha, \eta) \equiv 2+\frac{\eta_{0}}{\eta} \ln \alpha$.

It can be shown that:

i) $l(\alpha, \eta)$ is strictly increasing in $\eta$;

ii) $l\left(\alpha, \eta_{0}\right)=2$;

iii) $l\left(\alpha, 2 \eta_{0}\right)=2 \alpha^{1 / 2}$

iv) $m(\alpha, \eta)$ is strictly decreasing in $\eta$;

v) $m\left(\alpha, \eta_{0}\right)=2+\ln \alpha$

vi) $m\left(\alpha, 2 \eta_{0}\right)=2+\frac{1}{2} \ln \alpha$

vii) $m\left(\alpha, \eta_{0}\right)>l\left(\alpha, \eta_{0}\right)$, whose proof is trivial;

viii) $m\left(\alpha, 2 \eta_{0}\right)<l\left(\alpha, 2 \eta_{0}\right)$. Note that $m\left(\alpha, 2 \eta_{0}\right)<l\left(\alpha, 2 \eta_{0}\right) \Leftrightarrow n(\alpha) \equiv 2+\frac{1}{2} \ln \alpha$ $-2 \alpha^{1 / 2}<0$, and also that $n(1)=0$ and $n^{\prime}(\alpha)=\alpha^{-1 / 2}\left(\frac{1}{2 \alpha^{1 / 2}}-1\right)<0$ if $\alpha>1$. Therefore, if $\alpha>1$, then $n(\alpha)<0$; equivalently, $m\left(\alpha, 2 \eta_{0}\right)<l\left(\alpha, 2 \eta_{0}\right)$.

To sum up, from $i)$, iv), vii) and viii) one obtains that there is one unique $\hat{\eta} \in\left(\eta_{0}, 2 \eta_{0}\right)$ such that $l(\alpha, \hat{\eta})=m(\alpha, \hat{\eta})$, that is to say, $g(\alpha, \hat{\eta})=0$; equivalently, 
$K^{\prime}(\hat{\eta})=0$

Claim 6 Assume that $\beta<0, \alpha>1$ and $\eta_{0}>0$ : if $\eta>2 \eta_{0}$, then $K(\eta)$ is strictly decreasing.

Proof of Claim 6. Assume that $\beta<0, \alpha>1$ and $\eta_{0}>0$. From (45) one has that $K^{\prime}(\eta)=\frac{\eta_{0} \ln \alpha}{\beta \eta^{2}} g(\alpha, \eta)$, where $g(\alpha, \eta) \equiv 2 \alpha-\alpha^{\frac{\eta_{0}}{\eta}}\left(2+\frac{\eta_{0}}{\eta} \ln \alpha\right)$ is continuous in $\eta$ for all $\eta>0$. We have proved in Claim 4 that $K^{\prime}\left(2 \eta_{0}\right)<0$, that is, $g\left(\alpha, 2 \eta_{0}\right)>$ 0 . In addition, $\partial g(\alpha, \eta) / \partial \eta=\frac{\eta_{0}}{\eta^{2}} \alpha^{\frac{\eta_{0}}{\eta}}\left(3+\frac{\eta_{0}}{\eta} \ln \alpha\right) \ln \alpha>0$. Therefore, if $\eta>2 \eta_{0}$, then $g(\alpha, \eta)>0$ and $K^{\prime}(\eta)<0$.

Claim 7 If $\beta<0$ and $\alpha>1$, then $\lim _{\eta \rightarrow \infty} K(\eta)<0$.

Proof of Claim 7. Assume that $\beta<0$ and $\alpha>1$. From (45) one has that $\lim _{\eta \rightarrow \infty} K(\eta)=\frac{\alpha}{\beta}\left(\ln \alpha+\frac{1}{\alpha}-1\right) \equiv \frac{\alpha}{\beta} o(\alpha)$, where $o(\alpha) \equiv \ln \alpha+\frac{1}{\alpha}-1$. Therefore, $\lim _{\eta \rightarrow \infty} K(\eta)<0 \Leftrightarrow o(\alpha)>0$. Note that $o(1)=0$ and $o^{\prime}(\alpha)=\frac{1}{\alpha}\left(1-\frac{1}{\alpha}\right)>0$. Therefore, $\lim _{\eta \rightarrow \infty} K(\eta)<0$.

Claim 8 Assume $\beta<0, \alpha>1$ and $\eta_{0}>0$. Then i) there is a unique $\eta^{*}>2 \eta_{0}$ such that $K\left(\eta^{*}\right)=0$, and ii) $K(\eta)<0$ for all $\eta>\eta^{*}$.

Proof of Claim 8. From Claim 4, Claim 6 and Claim 7 the proof is trivial.

Now we are ready to prove Lemma 2 .

Proof of Lemma 2. From Claims 3-5 and Claim 8 the proof is trivial.

Proof of Proposition 1. From Lemma 1 and Lemma 2 the result is trivial.

Proof of Proposition 2. Assume that $\beta<0, \alpha>1$ and $\eta_{0}>0$. From Proposition 1 one has that there is at least one $x \in\left(0, T_{\max }(\eta)\right)$ such that $M(x, \eta)=0$. To prove that it is unique, we split $M(x, \eta)$ as the difference of two functions $p(x, \eta)$ and $q(x, \eta)$, and prove that one unique intersection point exists between them.

i) $M(x, \eta)$ can be rewritten as $M(x, \eta) \equiv p(x, \eta)-q(x, \eta)$, where $p(x, \eta) \equiv$ $2 \alpha x-\frac{1-\vartheta}{\beta} e^{-\beta \frac{\eta}{\eta_{0}} x}-\frac{\vartheta}{\beta} e^{-\beta J}$, and $q(x, \eta) \equiv x e^{-\beta x}+\vartheta \alpha J+(1-\vartheta) \alpha x \frac{\eta}{\eta_{0}}-\frac{1}{\beta} e^{-\beta x}$;

ii) using the definition of $J, p(0, \eta)=-\frac{1}{\beta}-\frac{\vartheta}{\beta}(\alpha-1)>0$;

iii) given the definitions of $J$ and $T_{\max }(\eta), p\left[T_{\max }(\eta), \eta\right]=-\frac{\alpha}{\beta}\left(2 \frac{\eta_{0}}{\eta} \ln \alpha+1\right)>0$;

iv) $\partial p(x, \eta) / \partial x=2 \alpha+\frac{(1-\vartheta) \eta}{\eta_{0}} e^{-\beta \frac{\eta}{\eta_{0}} x}>0$, that is, $p(x, \eta)$ is strictly increasing in $x ; v) \partial^{2} p(x, \eta) / \partial x^{2}=-\frac{(1-\vartheta) \eta^{2} \beta}{\eta_{0}^{2}} e^{-\beta \frac{\eta}{\eta_{0}} x}>0$, that is, $p(x, \eta)$ is strictly conve $x$ in $x$;

vi) $q(0, \eta) \equiv-\frac{1}{\beta}(\vartheta \alpha \ln \alpha+1)>0$ and $\alpha>1$;

vii) given the definitions of $J$ and $T_{\max }(\eta)$, 
$q\left[T_{\max }(\eta), \eta\right]=-\frac{1}{\beta}\left[\left(\alpha+\frac{\eta_{0}}{\eta} \alpha^{\frac{\eta_{0}}{\eta}}\right) \ln \alpha+\alpha^{\frac{\eta_{0}}{\eta}}\right]>0 ;$

viii) $\partial q(x, \eta) / \partial x=e^{-\beta x}(2-x \beta)+(1-\vartheta) \alpha \frac{\eta}{\eta_{0}}>0$, that is, $q(x, \eta)$ is strictly increasing in $x$;

ix) $\partial^{2} q(x, \eta) / \partial x^{2}=e^{-\beta x}\left(x \beta^{2}-3 \beta\right)>0$, that is, $q(x, \eta)$ is strictly convex in $x$;

x) $p(0, \eta)<q(0, \eta) \Leftrightarrow \alpha \ln \alpha-\alpha+1>0$; see expression $o(\alpha)$ in Claim 7;

xi) $p\left[T_{\max }(\eta), \eta\right]>q\left[T_{\max }(\eta), \eta\right] \Leftrightarrow M\left[T_{\max }(\eta), \eta\right] \equiv K(\eta)>0$ if $\eta \in\left(\eta_{0}, \eta^{*}\right)$. [See Lemma 2.]

Therefore, $p(x, \eta)$ and $q(x, \eta)$ cross each other only once between $x=0$ and $x=$ $T_{\max }(\eta)$, that is, there exists one unique $x \in\left(0, T_{\max }(\eta)\right)$ such that $p(x, \eta)=q(x, \eta)$ $\Leftrightarrow M(x, \eta)=0$. The following plot in Figure 7 can help us understand the proof.

\section{[INSERT FIGURE 8 AROUND HERE]}

Proof of Proposition 3. Given (1), $x=T$ is a solution to (37) if and only if $\widehat{M}(x, \eta)=0$, where

$$
\begin{gathered}
\widehat{M}(x, \eta) \equiv x\left(\alpha-e^{-\beta x}\right)+\alpha\left[x-\min \left\{\frac{\eta x}{\eta_{0}}, J\right\}\right] \\
+\frac{e^{-\beta x}-e^{-\beta \min \left\{\frac{\eta x}{\eta_{0}}, J\right\}}}{\beta}+\vartheta \alpha\left[\min \left\{\frac{\eta x}{\eta_{0}}, J\right\}-J\right]+\frac{\vartheta\left[e^{-\beta \min \left\{\frac{\eta x}{\eta_{0}}, J\right\}}-e^{-\beta J}\right]}{\beta} .
\end{gathered}
$$

The strategy of the proof will follow these steps:

i) first, we will prove that $\widehat{M}(J, \eta)=0$;

ii) second, we will prove that for values of $x$ less than and close enough to $J$ we will have that $\widehat{M}(x, \eta)>0$;

iii) third, we will prove that $\widehat{M}(0, \eta)<0$;

$i v$ ) fourth, given that $\widehat{M}(x, \eta)$ is continuous, at least one $x \in(0, J)$ exists such that $\widehat{M}(x, \eta)=0$; and

$v$ ) fifth, if such an $x$ is unique and interior, $0<x<T_{\max }(\eta)<J$, then $K(\eta)>0$, which implies that $\eta \in\left(\eta_{0}, \eta_{*}\right)$ as we have proven in Lemma 2 .

In subsection 3.3.1 we have proven that if the solution is interior, then it must be the case that $\eta>\eta_{0}$.

i) If so, $\min \left\{\frac{\eta J}{\eta_{0}}, J\right\}=J$. Therefore, from (57) and (2) one has that $\widehat{M}(J, \eta)=$ $J\left(\alpha-e^{-\beta J}\right)=0$. 
ii) Similarly, for $x$ less than but close enough to $J, \min \left\{\frac{\eta x}{\eta_{0}}, J\right\}=J$. Therefore, from (57) one has that

$$
\begin{aligned}
\widehat{M}(x, \eta) & \equiv x\left(\alpha-e^{-\beta x}\right)+\alpha(x-J)+\frac{e^{-\beta x}-e^{-\beta J}}{\beta} \\
& \Rightarrow \frac{\partial \widehat{M}(x, \eta)}{\partial x}=2\left(\alpha-e^{-\beta x}\right)+\beta x e^{-\beta x} \\
& \Rightarrow \lim _{x \rightarrow J-} \frac{\partial \widehat{M}(x, \eta)}{\partial x}=2\left(\alpha-e^{-\beta J}\right)+\beta x e^{-\beta J}=-\alpha \ln \alpha<0,
\end{aligned}
$$

given that $\alpha>1$. Thus, given $i$ ), for $x$ less than but close enough to $J$ one has that $\widehat{M}(x, \eta)>0$.

iii) From (57) one obtains that

$$
\widehat{M}(0, \eta)=\frac{\vartheta}{\beta}[1+\alpha(\ln \alpha-1)]<0 .
$$

The previous inequality is immediately checked if we assume that $\beta<0$ and define $f(\alpha) \equiv 1+\alpha(\ln \alpha-1)$, so that $f(1)=0, f^{\prime}(\alpha)=\ln \alpha>0$ and, therefore, $f(\alpha)>0$ for $\alpha>1$.

$i v)$ Thus, given that $\widehat{M}(x, \eta)$ is continuous, from $i i)$ and $i i i)$ one has that at least one $x^{*} \in(0, J)$ exists such that $\widehat{M}(x, \eta)=0$;

$v$ ) Given (43), from (44), (45) and (57) it can be obtained

$$
\widehat{M}\left[T_{\max }(\eta), \eta\right]=M\left[T_{\max }(\eta), \eta\right] \equiv K(\eta) .
$$

If the answer of item $i v)$ is unique and interior, that is to say, $0<x^{*}<T_{\max }(\eta)<J$, then $\widehat{M}\left[T_{\max }(\eta), \eta\right]>0$. In this case, given the equality in the previous expression, $K(\eta)>0$ and for this to happen we have seen that it is necessary that $\eta \in\left(\eta_{0}, \eta^{*}\right)$. [See Lemma2.]

Proof of Proposition 4. The strategy of the proof is as follows:

$i$ ) first, we prove that $T=T_{\max }\left(\eta^{*}\right) \equiv \frac{J \eta_{0}}{\eta^{*}}$ and $R=J$ is a solution to (39) and $(41)$;

ii) second, we prove that it is the unique one which meets the condition $0<T<$ $J$.

i) Assume that $\beta<0, \alpha>1, \eta=\eta_{*}>\eta_{0}>0$. From Lemma 2 one has that $K\left(\eta_{*}\right)=0$. Given the definition of $K(\eta) \equiv M\left[T_{\max }(\eta), \eta\right]$, one has that $M\left[T_{\max }\left(\eta_{*}\right), \eta_{*}\right]=0$; equivalently, $T_{\max }\left(\eta_{*}\right) \equiv \frac{J \eta_{0}}{\eta^{*}}<J$ is a solution to (41). In this case, from (39) one has that $R=J$. In sum, $T=T_{\max }\left(\eta_{*}\right) \equiv \frac{J \eta_{0}}{\eta^{*}}$ and $R=J$ satisfy (39) and (41), so that corner and interior solutions coincide. 
ii) To see that it is the unique one such that $0<T<J$ we have to prove that $T m(T)=\int_{T}^{R} m(\tau) d \tau+\vartheta \int_{R}^{J} m(\tau) d \tau=\int_{T}^{J} m(\tau) d \tau$ has one unique solution $T<J$ [because, trivially, $J m(J)=\int_{J}^{J} m(\tau) d \tau=0$ by definition of $J$ in (2)]. Given (1) and (2), it can be shown after some algebra that condition $\operatorname{Tm}(T)=\int_{T}^{J} m(\tau) d \tau$ is equivalent to $r(T, \alpha, \beta)=0$, where $r(T, \alpha, \beta) \equiv\left(T-\frac{1}{\beta}\right)\left(\alpha-e^{-\beta T}\right)-\alpha(J-T)$. Note that

ii.1) $r(T, \alpha, \beta)$ is continuous in $T$ and in $\alpha$;

ii.2) by definition of $J, r(0, \alpha, \beta)=\frac{-1}{\beta}(\alpha-1-\alpha \ln \alpha)$;

ii.3) $r(0,1, \beta)=0$;

ii.4) $\partial r(0, \alpha, \beta) / \partial \alpha=\frac{\ln \alpha}{\beta}<0$ if $\alpha>1$ and $\beta<0$ and that, therefore,

ii.5) $r(0, \alpha, \beta)<0$ if $\beta<0$ and $\alpha>1$.

ii.6) Given the definition of $J, r(J, \alpha, \beta)=0$.

ii.7) $\partial r(T, \alpha, \beta) / \partial T=2 \alpha-(2-\beta T) e^{-\beta T}$ and, therefore,

ii. 8$)$ if $T=0$, then $\partial r(T, \alpha, \beta) / \partial T=2(\alpha-1)>0$ if $\alpha>1$, and

ii.9) if $T=J$, then $\partial r(T, \alpha, \beta) / \partial T=-\alpha \ln \alpha<0$ if $\alpha>1$.

ii.10) To sum up, from ii.1), ii.5), ii.6), ii.8), and ii.9), one has that at least one $d \in(0, J)$ exists such that $\partial r(T, \alpha, \beta) / \partial T=0$ for $T=d$.

ii.11) Finally, it can be shown that $\partial^{2} r(T, \alpha, \beta) / \partial T^{2}=e^{-\beta T} \beta(3-\beta T)<0$ if $\beta<0$, that is, $r(T, \alpha, \beta)$ is strictly concave in $T$.

ii.12) Therefore, from $i i .10)$ and $i i .11)$ one has that there is one unique $d \in(0, J)$ for which $\partial r(T, \alpha, \beta) / \partial T=0$ for $T=d$ and, therefore,

ii.13) from ii.1), ii.5), ii.6) and ii.12) one has that one unique $T$ (where $0<$ $T<d<J]$ exists such that $r(T, \alpha, \beta)=0 \Leftrightarrow T m(T)=\int_{T}^{J} m(\tau) d \tau$.

\section{[INSERT FIGURE 9 AROUND HERE]}

Proof of Proposition 5. This is the strategy of the proof:

$i$ ) first, we will show that there is no $x \in\left[0, T_{\max }(\eta)\right]$ such that $(37)$ has solution or that, equivalently, $\widehat{M}(x, \eta)$ defined in (57) takes the value zero.

ii) second, we will show that $\widehat{M}(J, \eta)=0$, therefore $J$ is a solution.

iii) third, we will prove that one unique $x \in\left(T_{\max }(\eta), J\right)$ exists such that $\widehat{M}(x, \eta)=0$, where $x=T_{\max }\left(\eta_{*}\right) \equiv \frac{J \eta_{0}}{\eta_{*}}$, so that $T=T_{\max }\left(\eta_{*}\right) \equiv \frac{J \eta_{0}}{\eta_{*}}$, and $R$ $=J$. 
i.1) Assume that $\beta<0, \alpha>1$ and $\eta>\eta_{*}>\eta_{0}>0$. Given (43), from (44), (45) and (57) it can be checked that

$$
\widehat{M}\left[T_{\max }(\eta), \eta\right]=M\left[T_{\max }(\eta), \eta\right] \equiv K(\eta)
$$

According to Claim 8, if $\eta>\eta_{*}$, then $K(\eta)<0$. Therefore, $\widehat{M}\left[T_{\max }(\eta), \eta\right]<0$.

i.2) $\widehat{M}(x, \eta)$ defined in (57) can be rewritten as $\widehat{M}(x, \eta)=\hat{p}(x, \eta)-\hat{q}(x, \eta)$, where

$$
\begin{gathered}
\hat{p}(x, \eta) \equiv 2 \alpha x-\frac{(1-\vartheta)}{\beta} e^{-\beta \min \left\{\frac{\eta x}{\eta_{0}}, J\right\}}-\frac{\vartheta}{\beta} e^{-\beta J}, \quad \text { and } \\
\hat{q}(x, \eta) \equiv x e^{-\beta x}+\alpha \vartheta J+\alpha(1-\vartheta) \min \left\{\frac{\eta x}{\eta_{0}}, J\right\}-\frac{e^{-\beta x}}{\beta} .
\end{gathered}
$$

i.3) Let us assume values of $x \in\left[0, T_{\max }(\eta)\right]$. In this case, $\min \left\{\frac{\eta x}{\eta_{0}}, J\right\}=\frac{\eta x}{\eta_{0}}$, so that

$$
\begin{aligned}
& \hat{p}(x, \eta) \equiv 2 \alpha x-\frac{(1-\vartheta)}{\beta} e^{-\beta \frac{\eta x}{\eta_{0}}}-\frac{\vartheta}{\beta} e^{-\beta J}, \quad \text { and } \\
& \hat{q}(x, \eta) \equiv x e^{-\beta x}+\alpha \vartheta J+\alpha(1-\vartheta) \frac{\eta x}{\eta_{0}}-\frac{e^{-\beta x}}{\beta} .
\end{aligned}
$$

i.4) $\hat{p}(0, \eta)=\frac{-1}{\beta}[1+\vartheta(\alpha-1)]>0$.

i.5) $\hat{p}\left[T_{\max }(\eta), \eta\right]=\frac{-\alpha}{\beta}\left[1+2 \frac{\eta_{0}}{\eta} \ln \alpha\right]>0$.

i.6) $\frac{\partial \hat{p}(x, \eta)}{\partial x}=2 \alpha+\frac{(1-\vartheta) \eta}{\eta_{0}} e^{-\beta \frac{\eta x}{\eta_{0}}}>0: \hat{p}(x, \eta)$ is strictly increasing for $x \in$ $\left(0, T_{\max }(\eta)\right)$.

$i .7) \frac{\partial^{2} \hat{p}(x, \eta)}{\partial x^{2}}=\frac{-(1-\vartheta) \beta \eta^{2}}{\eta_{0}^{2}} e^{-\beta \frac{\eta x}{\eta_{0}}}>0: \hat{p}(x, \eta)$ is strictly convex for $x \in\left(0, T_{\max }(\eta)\right)$.

i.8) $\hat{q}(0, \eta)=\frac{-1}{\beta}[1+\alpha \vartheta \ln \alpha]>0$.

i.g) $\hat{q}\left[T_{\max }(\eta), \eta\right]=\frac{-1}{\beta}\left[\alpha^{\frac{\eta_{0}}{\eta}}+\left(\alpha+\frac{\eta_{0}}{\eta} \alpha^{\frac{\eta_{0}}{\eta}}\right) \ln \alpha\right]>0$.

i.10) $\frac{\partial \hat{q}(x, \eta)}{\partial x}=(2-\beta x) e^{-\beta x}+\alpha(1-\vartheta) \frac{\eta}{\eta_{0}}>0: \hat{q}(x, \eta)$ is strictly increasing for $x \in\left(0, T_{\max }(\eta)\right)$.

i.11) $\frac{\partial^{2} \hat{q}(x, \eta)}{\partial x^{2}}=\left(x \beta^{2}-3 \beta\right) e^{-\beta x}: \hat{q}(x, \eta)$ is strictly convex for $x \in\left(0, T_{\max }(\eta)\right)$.

i.12) From i.1) and $i .8)$ one has that $\hat{p}(0, \eta)-\hat{q}(0, \eta)=-1+\alpha(1-\ln \alpha)<0$.

i.13) From i.1) and i.2) ones has that $\widehat{M}\left[T_{\max }(\eta), \eta\right] \equiv \hat{p}\left[T_{\max }(\eta), \eta\right]-\hat{q}\left[T_{\max }(\eta), \eta\right]$ $<0$.

i.14) Therefore, from $i .4)-i .13)$ one has that $\hat{p}(x, \eta)$ and $\hat{q}(x, \eta)$ do not cross each other at any $x \in\left[0, T_{\max }(\eta)\right]$; equivalently, no $x \in\left[0, T_{\max }(\eta)\right]$ exists such that $\hat{M}(x, \eta)=0$. And if there is some $x$ for which $\hat{M}(x, \eta)=0$, then $x \in\left(T_{\max }(\eta), J\right]$.

ii) Given that $\eta>\eta_{*}>\eta_{0}, \min \left\{\frac{\eta J}{\eta_{0}}, J\right\}=J$ and, therefore, from (57) one has that $\hat{M}(J, \eta)=0$, that is $x=J$ is a solution to (57). 
iii) We are going to prove that one unique $T \in\left(T_{\max }(\eta), J\right)$ exists such that $\widehat{M}(x, \eta)=0$, where $T=T_{\max }\left(\eta_{*}\right) \equiv \frac{J \eta_{0}}{\eta_{*}}$; this way $T>T_{\max }(\eta)$, and $R=J$.

iii.1) From $\widehat{M}(x, \eta)$ defined in (57), for $\eta>\eta_{0}$ and for $x$ close enough to $J$, one has that $\frac{\eta x}{\eta_{0}}>J$, so that $\min \left\{\frac{\eta x}{\eta_{0}}, J\right\}=J$. Therefore, for $x$ close enough to $J$,

$$
\begin{aligned}
\widehat{M}(x, \eta) & \equiv x\left(\alpha-e^{-\beta x}\right)+\alpha(x-J)+\frac{e^{-\beta x}-e^{-\beta J}}{\beta} \\
& \Rightarrow \frac{\partial \widehat{M}(x, \eta)}{\partial x}=2\left(\alpha-e^{-\beta x}\right)+\beta x e^{-\beta x} \\
& \Rightarrow \lim _{x \rightarrow J-} \frac{\partial \widehat{M}(x, \eta)}{\partial x}=\beta x e^{-\beta J}=-\alpha \ln \alpha<0 .
\end{aligned}
$$

Therefore, $\widehat{M}(x, \eta)>0$ for $x$ close enough to $J$. Thus, there exists at least one $T$ $\in\left(T_{\max }(\eta), J\right)$ such that $\widehat{M}(T, \eta)=0$. If $T>T_{\max }(\eta)$, then $T>\frac{J \eta_{0}}{\eta} \Leftrightarrow \frac{T \eta}{\eta_{0}}>J \Rightarrow$ $R=\min \left\{\frac{T \eta}{\eta_{0}}, J\right\}=J$.

But if so, from (36) and (37) one has that $T$ must satisfy

$$
\operatorname{Tm}(T)=\int_{T}^{J} m(\tau) d \tau .
$$

And this is, precisely, Case $i$ ) studied in Proposition 4. Therefore, one unique $T<J$ exists which satisfies the previous equation, so that $T=T_{\max }\left(\eta_{*}\right) \equiv \frac{J \eta_{0}}{\eta^{*}}<J$ if $\eta_{*}>\eta_{0}$. Note that, additionally, $T>T_{\max }(\eta)$ because by definition of $T_{\max }(\eta)$ in (43), $\frac{J \eta_{0}}{\eta_{*}}>\frac{J \eta_{0}}{\eta} \Leftrightarrow \eta>\eta_{*}$. In short, if $\eta>\eta_{*}$, then $T=T_{\max }\left(\eta_{*}\right)$ and $R=J$.

Proof of Proposition 6. If $\eta=\eta_{0}$, from (36) one has that $R=T \leq J$, because $T$ cannot be greater than $J$. At any rate, $R=T$, so that social security tax revenues are zero. Social security budget balance is required so that $\vartheta=0$. From (47) and (48) we obtain that

$$
\operatorname{Tm}(T)=0 .
$$

The strategy of the proof consists of two steps: first, we prove that (58) admits only two solutions; and, second, we prove that the indirect utility function attains a higher value in one of the two.

Equation (58) has 2 possible solutions: $T=0$ and $T=J$. To check that $\operatorname{Tm}(T)=0$ admits only these 2 solutions, notice that $\operatorname{Tm}(T)=0 \Leftrightarrow u(T) \equiv$ $T\left(\alpha-e^{-\beta T}\right)=0$.

i) $u(0)=0: 0$ is a solution;

ii) $u(J)=0: J$ is a solution; 
iii) $u^{\prime}(T)=e^{-\beta T}(\beta T-1)+\alpha$;

iv) $u^{\prime}(0)=\alpha-1>0(u$ is increasing in $T=0)$;

v) $u^{\prime}(J)=-\ln \alpha<0(u$ is decreasing in $T=J)$; and

vi) $u^{\prime \prime}(T)=\beta e^{-\beta T}(2-\beta T)<0[i . e ., u(T)$ is strictly concave and, therefore, no $T \in(0, J)$ exists which is a solution to (58)].

To sum up, there are two solutions: $0=T=R<J$, and $0<T=R=J$. The situation is described in Figure 10.

\section{[INSERT FIGURE 10 AROUND HERE]}

Finally, we will prove that the indirect utility function is higher at $T=0$ than at $T=J$.

i) Substituting the rest of equality restrictions into the first restriction of (20);

ii) taking into account that $\omega(\tau)=\bar{\omega}(t)=\omega, T(t)=T, R(t)=\eta T / \eta_{0}$, and that $m(\tau-t)=D(t, \tau)[$ given $(1)]$

iii) solving for $\int_{t}^{t+J} C(t, \tau) m(\tau-t) d \tau$ and substituting into the lifetime utility function (14);

$i v$ ) recalling that $\eta \equiv \mu \phi$, we obtain the indirect utility function for a solution in which $R=T \eta / \eta_{0}$ (without loss of generality, we assume an individual born at $t=0)$, and

$v$ ) assuming $\eta=\eta_{0}$ and $\vartheta=0$, we obtain

$$
V(T, \eta)=-\frac{\bar{H}}{\phi} \int_{0}^{T} \tau\left(\frac{e^{-\beta \tau}-\alpha}{1-\alpha}\right) d \tau,
$$

which, trivially, is decreasing in $T$. Therefore, solution $0=T=R<J$ is preferred to solution $0<T=R=J$.

Proof of Proposition 8. The proof is trivial. (54) can be rewritten as

$$
\int_{T}^{R} e^{-\gamma z} f(z) d z=\frac{\kappa(\alpha-1)}{\mu T},
$$

where $f(z) \equiv e^{-n z}\left(\alpha-e^{-\beta z}\right)>0$ if $z<J$. Denoting the left-hand-side of (59) by $I(\gamma)$ and differentiating with respect to $\gamma$, one has

$$
\frac{d I(\gamma)}{d \gamma}=-\int_{T}^{R} e^{-\gamma z} z f(z) d z<0,
$$

if $T<R$, i.e., $I(\gamma)$ is strictly decreasing in $\gamma$. Therefore, at most one unique $\gamma$ exists which satisfies (59).

Proof of Proposition 9. From (1) and (55) this turns out to be trivial. 


\section{Acknowledgements}

We wish to thank Fundación BBVA, through Project 1/BBVA 00044.321 15467/2002, Ministry of Science and Technology, through Project SEC2003 - 04826

/ ECO, and Universidad del País Vasco through Grupo de Investigación Consolidado 9/UPV 00035.321 - 13511 / 2001 for their financial support. 


\section{REFERENCES}

Attanasio, O., Low, Hamish and Sánchez-Marcos, V., 2004. Explaining changes in female labor supply in a life-cycle model. University of Cambridge Working Papers 2004, 04/51.

Azariadis, C. , Drazen, A., 1990. Threshold externalities in economic development. Quarterly Journal of Economics 105, 501-526.

Barro, R., Sala i Martín, X., 1995. Economic Growth, 3 Ed, McGraw Hill, New York.

Barro, R., Lee, J-W., 2000. International data on educational attainment: updates and implications.Center for International Development at Harvard University Working Paper No. 42, April.

Bassanini. A., Scarpetta, S., 2001. Does human capital matter for growth in OECD countries? Evidence from pooled mean-group estimates. Economics Department Working Papers 282. ECO/WKP (2001) 8, OECD.

Benabou, R., 1993. Workings of a City: Location, education, and production. Quarterly Journal of Economics 108(3), 619-652.

Blanchard, O. J., 1985. Debts, deficits and finite horizons. Journal of Political Economy 93, 2, 223-247.

Boldrin, M., Jiménez-Martín, S., Perachi, F., 1997. Social security and retirement in Spain. NBER Working Paper Series Working Paper 6136.

Boucekkine, R., de la Croix, D., Licandro, O., 2002. Vintage human capital, demographic trends and endogenous growth. Journal of Economic Theory 104, 340375 .

Butcher, K. F., Case, A., 1994. The effect of sibling composition on women's education and earnings. Quarterly Journal of Economics 109, 531-563.

Coile, C., Gruber, J., 2000. Social security and retirement. NBER Working Paper Series Working Paper 7830.

Coronado, J. L., Fullerton, D., Glass, T., 2000. The progressivity of social security. NBER Working Paper Series Working Paper 7520.

De la Croix, D., Licandro, O., 1999. Life expectancy and endogenous growth. Economics Letters 65, 255-263.

Diamond, P., Gruber, J., 1997. Social security and retirement in the U.S.. NBER Working Paper Series, W.P. 6097.

Echevarría, C. A., 2003. Life expectancy, retirement and endogenous growth. Economic Modelling 21, 147-174. 
Echevarría, C. A., 2004. Life expectancy, schooling time, retirement and growth. Economic Inquiry 42(4), 602-617.

Ehrlich, I., Lui, F. T., 1991. Intergenerational trade, longevity and economic growth. Journal of Political Economy, October, 1029-1059.

Einarsson, T., Marquis, M. H. , 1996. Note on human capital externalities. Journal of Macroeconomics, Spring, Vol.18, No. 2, 341-351.

Fullerton, H. N. Jr., 1999. Labor force participation: 75 years of change, 1950 and 1998-2025. Monthly Labor Review, 122(12), December, 3-12.

Fuster, L., 1999. Effects of uncertain lifetime and annuity insurance on capital accumulation and growth. Economic Theory 13, 429-445.

Gendell, M., 1998. Trends in retirement age in four countries, 1965-95. Monthly Labor Review, August, 20-30.

Gittleman, M., ten Raa, T., Wolff, E. N., 2003. The vintage effect in TFP growth: an analysis of the age structure of capital. NBER, Working Paper 9768.

$\mathrm{Hu}$, S., 1999. Economic growth in the perpetual-youth model: implications of the annuity market and demographics. Journal of Macroeconomics, Winter 21(1), 107-124.

Jensen, J. B., McGuckin, R. H., Stiroh, K. J., 2001. The impact of vintage and survival on productivity: evidence from cohorts of U.S. manufacturing plants. Review of Economics and Statistics, May, 83(2), 323-332.

Jiménez-Martín, S., Sánchez, A. R., 1999. Incentivos y reglas de jubilación en España. Información Comercial Española, Cuadernos Económicos, 65, 45-88.

Kalemli-Ozcan, S., 2002a. Mortality change, the uncertainty effect, and retirement. NBER Working Paper Series, WP 8742.

Kalemli-Ozcan, S., 2002b. Does the mortality decline promote economic growth?. Journal of Economic Growth 7, 4-11-439.

Kelley, A., Schmidt, R., 1995. Aggregate population and economic growth correlations: the role of the components of demographic changes, Demography 32, 543-555.

Kotlikoff, L. J., Smetters, K., Walliser, J., 1999. Privatizing social security in the United States - Comparing the options. Review of Economic Dynamics 2, 532-574.

Lucas, R. E. Jr., 1988. On the mechanics of economic development. Journal of Monetary Economics, 22, 3-42.

Lucas, R. E. Jr., 1990. Supply-side economics: an analytical review. Oxford Economic Papers, 42, 293-316.

Maddison, A., 1995. Monitoring the world economy 1820-1992. Development 
Centre Studies, OECD, Paris.

Malmberg, B., 1994. Age structure effects on economic growth. Swedish evidence. Scandinavian Economic History Review, 42-3, 279-295.

Miles, D., 1999. Modelling the impact of demographic change upon the economy. The Economic Journal 109, January, 1-36.

Nerlove, M., Razin, A., Sadka, E. , von Weizsäcker, R., 1993. Comprehensive income taxation, investments in human and physical capital, and productivity. Journal of Public Economics 50, 397-406.

Neuman, S., Weiss, A., 1995. On the effects of schooling vintage on experienceearnings profiles: theory and evidence. European Economic Review, May, 39(5), 943-955.

Rodriguez, F., Sachs, J. D., 1999. Why do resource-abundant economies grow more slowly?. Journal of Economic Growth 4, 277-303.

Sabatini Dwyer, D., Mitchell, O. S., 1999. Health problems as determinants of retirement: are self-rated measures endogenous?. Journal of Health Economics 18, 173-193.

Schoen, R., 1988. Modeling Multigroup Populations, New York: Plenum Press.

Stokey, N., Rebelo, S., 1995. Growth effects of flat rate taxes. Journal of Political Economy 30, 419-450.

Szafran, R. F., 2002. Age-adjusted labor force participation rates, 1960-2045. Monthly Labor Review, 125(9), September, 25-38.

Violante, G. L., 2002. Technological acceleration, skill transferability, and the rise in residual inequality. Quarterly Journal of Economics, February, 117(1), 297338.

World Health Organization, 1999. The World Health Report 1999: Making a Difference, Geneva, Switzerland.

Yaari, M. E., 1965. Uncertain lifetime, life insurance, and the theory of the consumer. Review of Economic Studies 32, 137-150.

Zhang, J., Zhang, J., 2003. Long-run effects of unfunded social security with earnings-dependent benefits. Journal of Economic Dynamics \& Control 28, 617641.

Zhang, J., Zhang, J., 2004. How does social security affect economic growth? Evidence from cross-country data. Journal of Population Economics 17(3), 473-500.

Zhang, J., Zhang, J., 2005. The effect of life expectancy on fertility, saving, schooling and economic growth: theory and evidence. Scandinavian Journal of Economics 107(1), 45-66. 
Zhang, J., Zhang, J., Lee, R., 2001. Mortality decline and long-run economic growth. Journal of Public Economics 80, 485-507.

Zhang, J., Zhang, J., Lee, R., 2003. Rising longevity, education, savings, and growth. Journal of Development Economics 70, 83-101. 


\section{Tables}

\begin{tabular}{lcccccccc}
\multicolumn{10}{c}{ TABLE $1:$} \\
\hline \hline CoUnTRY & $\alpha$ & $\beta$ & $n$ & $E V_{O}$ & $E V_{T}$ & $\hat{a}_{O}$ & $\hat{a}_{T}$ & $J$ \\
\hline Spain & 27.9 & -0.031 & 0.002 & 79.1 & 79.1 & 38.0 & 39.1 & 107.4 \\
France & 21.3 & -0.028 & 0.005 & 78.8 & 78.9 & 37.7 & 36.3 & 109.2 \\
Italy & 21.4 & -0.028 & 0.003 & 79.1 & 79.1 & 41.6 & 38.3 & 109.4 \\
Great Britain & 28.6 & -0.032 & 0.004 & 77.8 & 77.3 & 36.4 & 36.4 & 104.8 \\
Canada & 21.9 & -0.028 & 0.010 & 79.4 & 79.8 & 37.2 & 32.1 & 110.2 \\
United States & 29.7 & -0.033 & 0.010 & 76.6 & 76.0 & 35.8 & 38.6 & 102.8 \\
\hline
\end{tabular}

Key: $E V$, life expectancy; $\hat{a}$, median age; $J$, maximum age. Subindex "T" denotes theoretical value; and subindex "O" denotes observed. Source for $\hat{a}_{O}$ : UNECE Statistical Division United Nations Economic Commission for Europe, Table 1.1. Basic Population Data and Structures in 2001, http://www.unece.org/stats/trends/Ch1/1.xls. Figures correspond to 2001. Source for $E V_{O}$ and $n$ : US Census Bureau International Data Base, http://www.census.gov/ipc/www/idbsum.html. Figures correspond to 2000.

TABle 2: Interior VS. CORner SOlutions

\begin{tabular}{ccc}
\hline \hline $0<\eta \leq \eta_{0}$ & $\eta_{0}<\eta<\eta^{*}$ & $\eta \geq \eta^{*}$ \\
\hline $0=T=R<J$ & $0<T<R<J$ & $T=T_{\max }\left(\eta^{*}\right)=\frac{J \eta_{0}}{\eta^{*}}$ \\
& & $R=J$ \\
\hline
\end{tabular}

TABLE 3. BenChMARK CASE

\begin{tabular}{ll}
\hline \hline Demographics & \\
\hline Parameters & Results \\
$n=0.010$ (Id.), & $\hat{a}=31.65(35.9), \bar{a}=37.08(36.2)$, \\
$\beta=-0.017$, & $E V=77.93(77), 1 / \kappa=0.019(0.015)$, \\
$\alpha=7.5$ & $J=118.5$ \\
Non-demographics & \\
\hline Parameters with $S . S$. & Results \\
$s=0.135$ (Id.), $\mu=0.286$, & $T=34.5(12.33), R=60.0(61.6)$, \\
$\phi=0.485, \omega=24.302$ & $\gamma=1.97 \%(2.1 \%), \vartheta=0.39$ \\
\hline Parameters without $S . S$. & Results \\
$s=0, \mu=0.251$, & $T=28.6, R=60.0, \gamma=2.0 \%$ \\
$\phi=8.3075, \omega=1.006$ & \\
\hline
\end{tabular}


Key to Table 3. Observed values are shown in parentheses.

\begin{tabular}{llllllll}
\multicolumn{8}{c}{ TABLE 4. Without VS. WITH SOCIAL SECURITY } \\
\hline \hline Period & $E V$ & $T$ & $\gamma$ & $L F P_{A}$ & $L F P_{M}$ & $L F P_{M}^{y}$ & $L F P_{M}^{e}$ \\
\hline $1870-1890$ & 41.4 & 5.6 & 1.89 & 37.5 & n.a. & n.a. & n.a. \\
$1890-1910$ & 46.4 & 7.1 & 1.95 & 39.0 & n.a. & n.a. & n.a. \\
$1910-1940$ & 56.5 & 8.2 & 1.13 & 37.1 & n.a. & n.a. & n.a. \\
\hline $1950-1970$ & 69.5 & 8.9 & 2.23 & 59.8 & 83.1 & 73.4 & 36.3 \\
$1970-1990$ & 73.1 & 10.9 & 2.39 & 63.4 & 77.9 & 70.5 & 21.6 \\
$1990-2000$ & 76.2 & 12.1 & 2.32 & 66.8 & 75.4 & 71.7 & 17.0 \\
\hline
\end{tabular}

Key to Table 4. EV : life expectancy at birth; from 1870 to 1940, figures correspond to white males; from 1950 to 2000, figures correspond to total population. T: from 1870 to 1940, years of schooling weighted by education; from 1950 to 2000, average years of schooling. $\gamma$ : growth of per capita GDP (in \%). $L F P_{A}$ : from 1870 to 1940 figures correspond to the employed population to the total population ratio; from 1950 to 2000, aggregate labor force participation; $L F P_{M}$ : from 1950 to 2000, labor force participation among male workers; $L F P_{M}^{y}$ : from 1950 to 2000, labor force participation among young male workers (16-24 years); $L F P_{M}^{e}$ : from 1950 to 2000, labor force participation among elderly male workers (65 and older). n.a.: not available. 
Figures
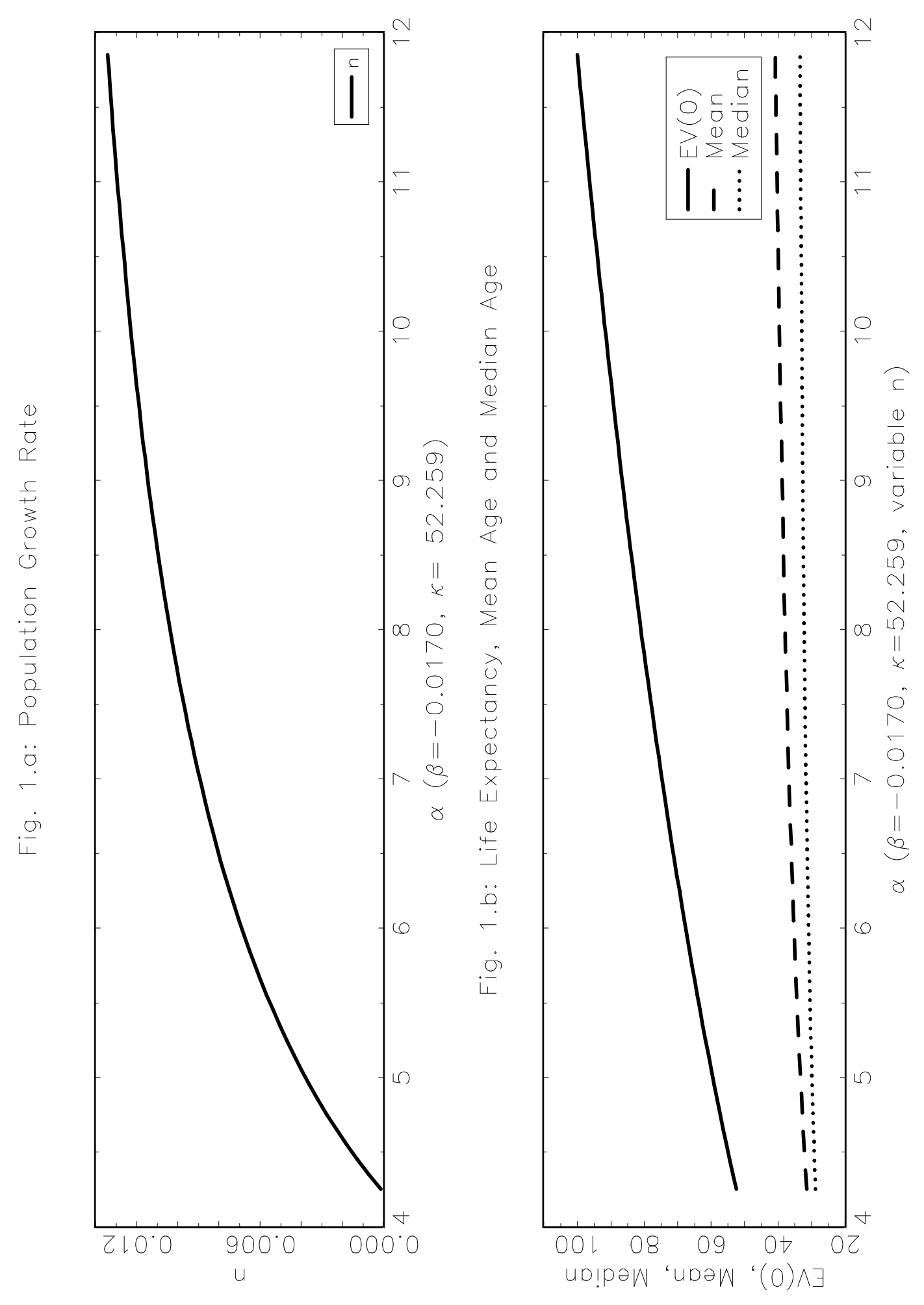


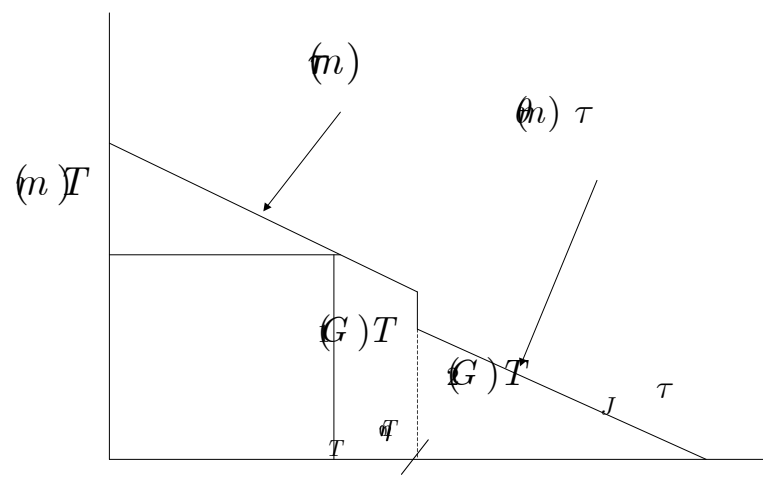

Figure 2

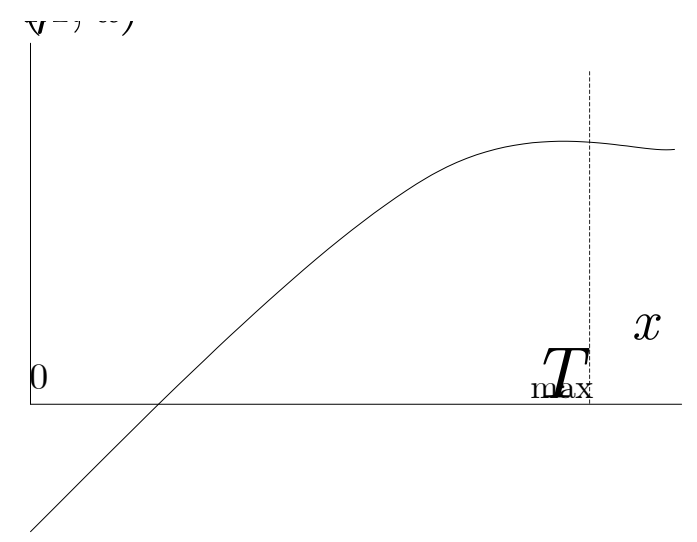

Figure 3

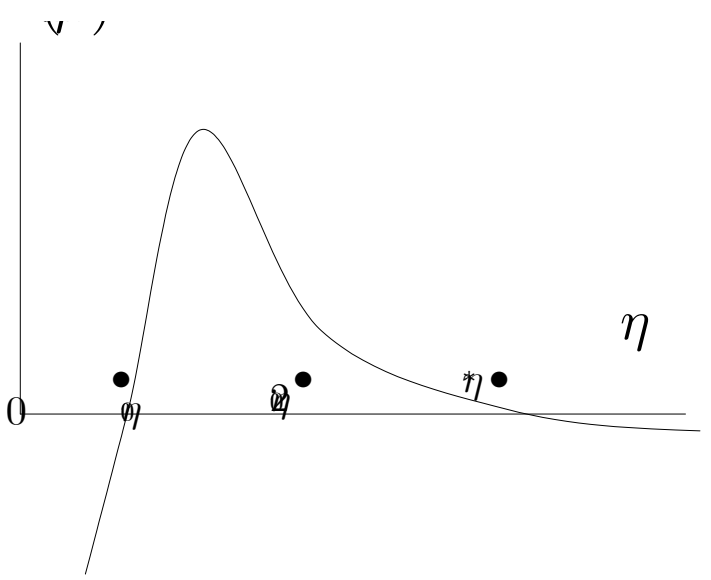

Figure 4 

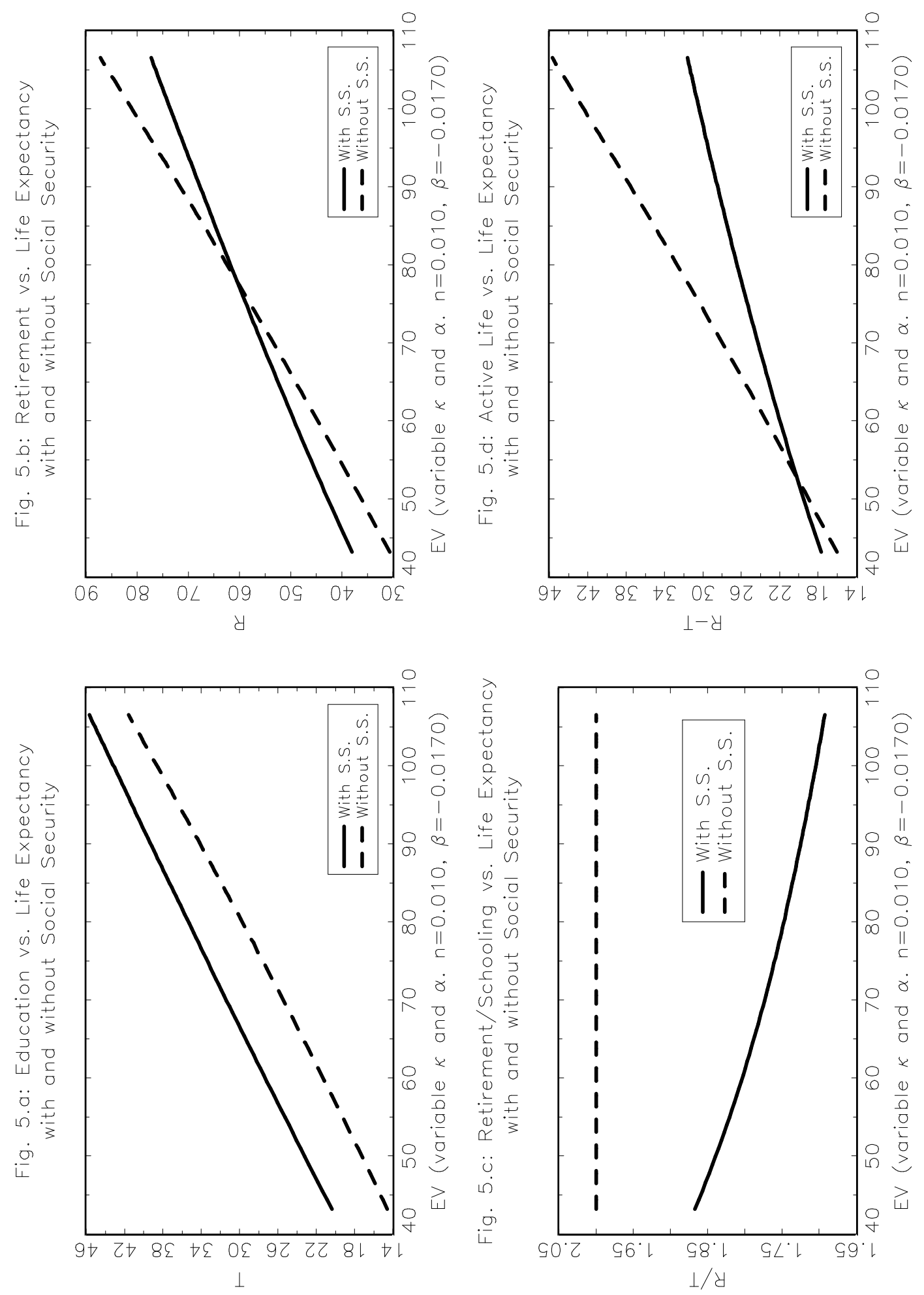

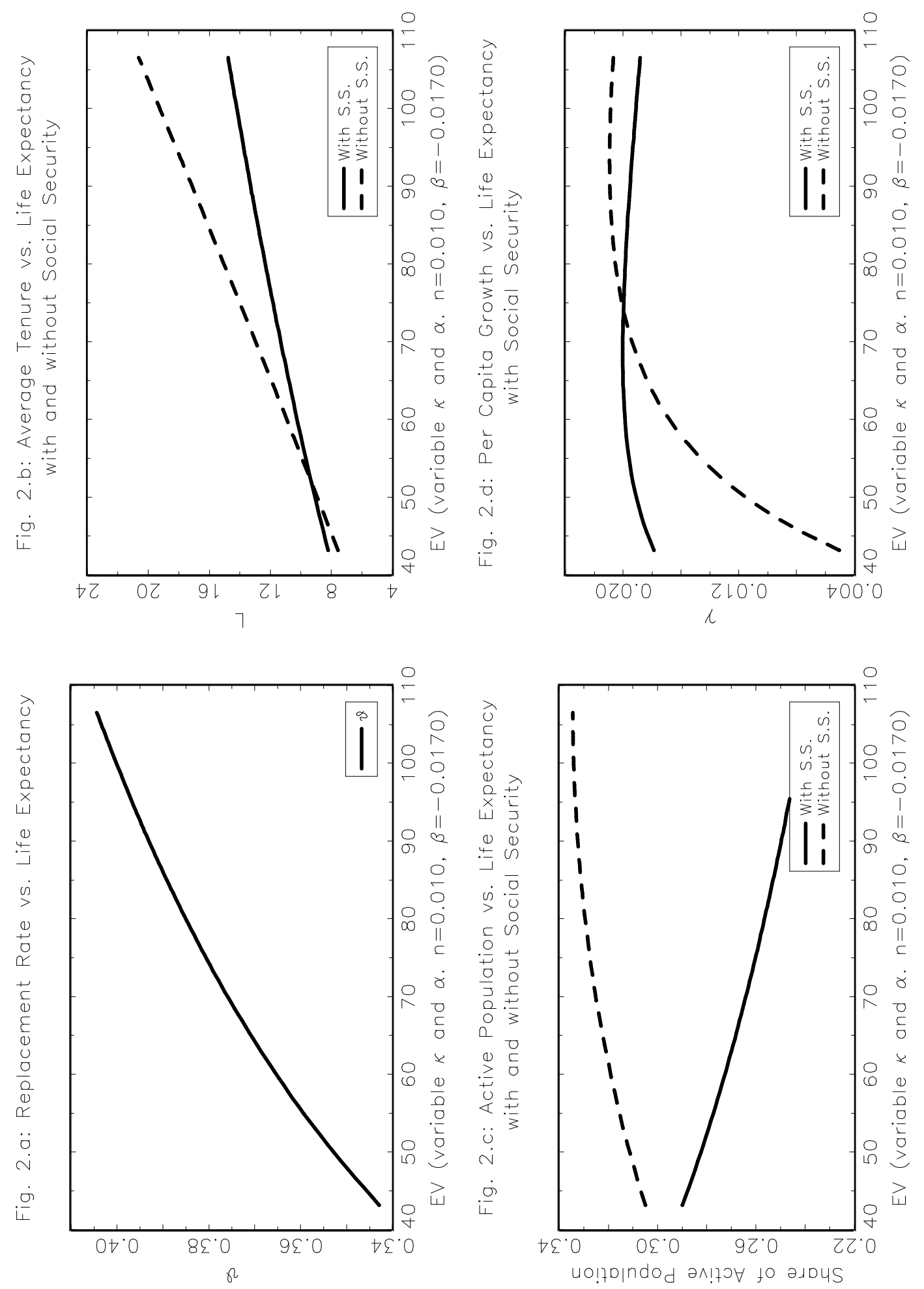

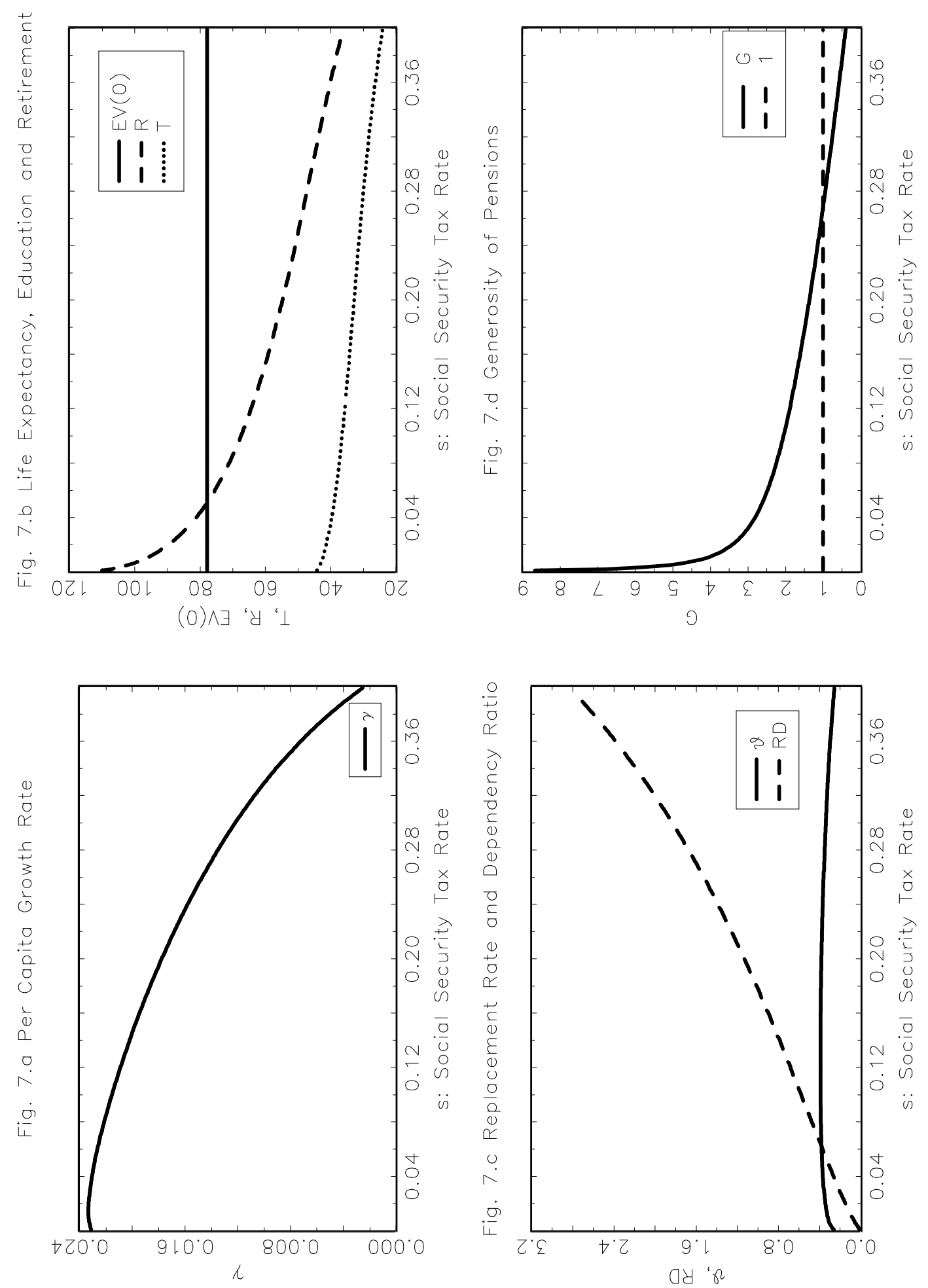


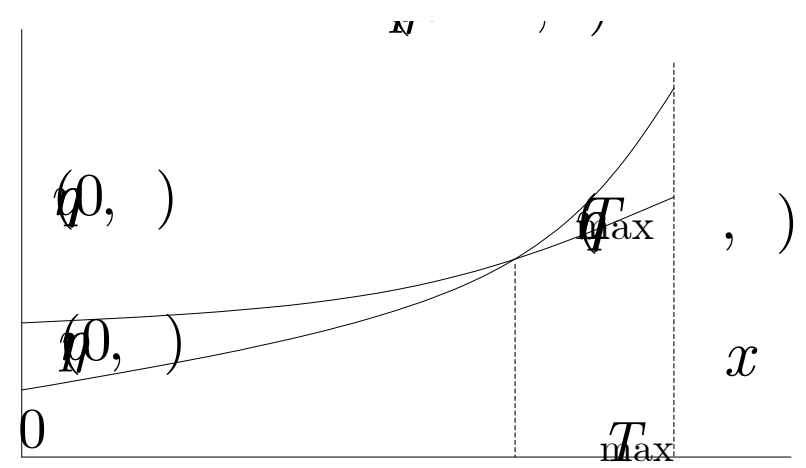

Figure 8

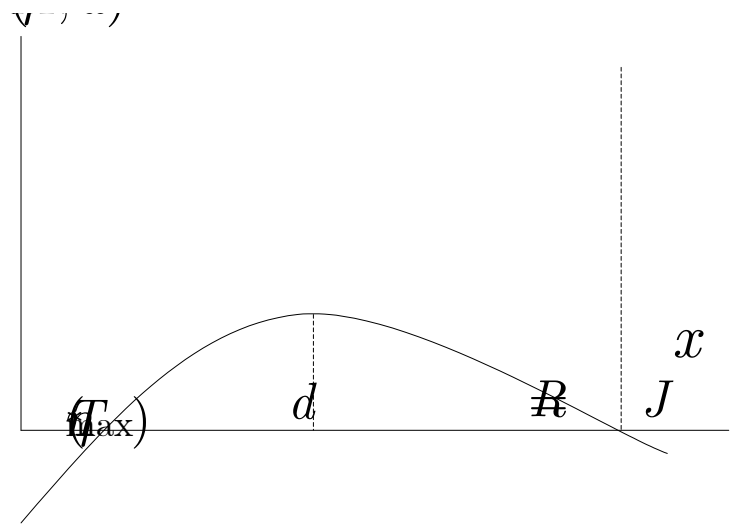

Figure 9

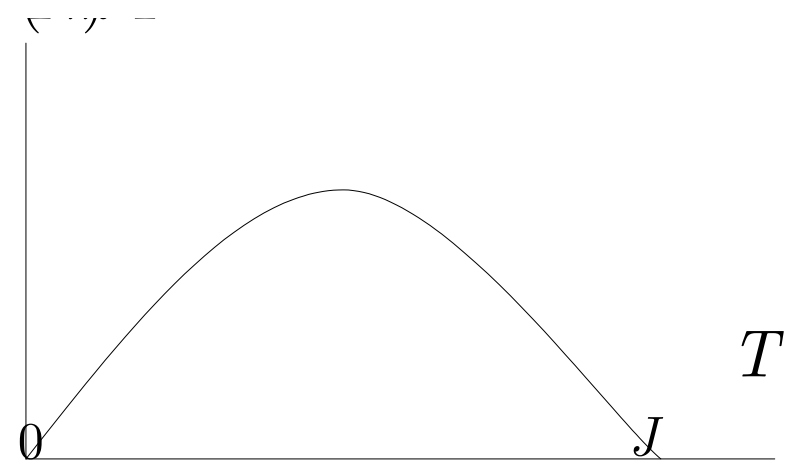

Figure 10 